\title{
PROPERTIES OF THE CHEMOSTAT MODEL WITH AGGREGATED BIOMASS AND DISTINCT REMOVAL RATES*
}

\author{
RADHOUANE FEKIH-SALEM ${ }^{\dagger}$ AND TEWFIK SARI ${ }^{\ddagger}$
}

\begin{abstract}
Understanding and exploiting the flocculation process is a major challenge in the mathematical theory of the chemostat. Here, we study a model of the chemostat involving the flocculating and deflocculating dynamics of planktonic and attached biomass competing for a single nutrient. In our study, the mortality (or maintenance) of species is taken into account and not neglected as in previous studies. The model is a three-dimensional system of ordinary differential equations. Using general monotonic functional responses, we give a complete analysis for the existence and local stability of all steady states. The theoretical analysis of the model involving the mortality is a difficult problem since the model is not reduced to a planar system as in the case where the dilution rates of the substrate and the biomass are equal.

With the same dilution rates, it is well known that the model can have a positive steady state which is unique and stable as long as it exists. Without mortality, and different dilution rates, the system may have a multiplicity of positive steady states that can only appear or disappear through saddle-node or transcritical bifurcations. In contrast to the case without mortality, under the joined effect of flocculation and mortality, the model may undergo supercritical Hopf bifurcations or homoclinic bifurcations, with the appearance or the disappearance of a stable periodic orbit. Therefore coexistence may occur around a positive steady state, and also around periodic oscillations.
\end{abstract}

Key words. Bi-stability, Chemostat, Flocculation, Limit cycles, Supercritical Hopf bifurcation

AMS subject classifications. 92B05, 34D20

1. Introduction. The chemostat plays an important role as a model in mathematical biology. In its simplest form, it is a model of a vessel where the populations compete for the available nutrient. It is used as the starting point for models of waste-water treatment processes. The derivation and analysis of a large number of chemostat-like models can be found in the monographs [33], see also [12]. We recall the classical chemostat model for a single species $x$ consuming a substrate $S$ :

$$
\left\{\begin{aligned}
\dot{S} & =D\left(S_{\text {in }}-S\right)-f(S) \frac{x}{\gamma} \\
\dot{x} & =[f(S)-D] x
\end{aligned}\right.
$$

where the dilution rate $D$ and the input concentration $S_{\text {in }}$ are the manipulated parameters, $f(S)$ is the growth function and $\gamma$ is the yield, which can be easily normalized to 1 , using the change of variable $x / \gamma \rightarrow x$. The growth function $f(S)$ is a non-negative Lipschitz continuous function with $f(0)=0$. Besides the washout steady state $E_{0}=\left(S_{i n}, 0\right)$, the system (1.1) can have a positive steady state $E^{*}=$ $\left(S^{*}, x^{*}\right)$, where $f\left(S^{*}\right)=D$ and $x^{*}=S_{i n}-S^{*}$. For monotonic $f(S)$, this steady state exists as long as $f\left(S_{i n}\right)>D$. It is unique and Locally Exponentially Stable (LES) as long as it exists. It is globally asymptotically stable in the quadrant $S \geq 0, x>0$. For non-monotonic growth function $f(S)$, the positive steady state is in general not unique and bi-stability may occur. When the function $f(S)$ is unknown and using the characterization $f\left(S^{*}\right)=D$ of a positive steady state, it is possible to reconstruct its graph using variations on $D$ and on-line measurements for the variable $S$. This problem of kinetics estimation in biological and biochemical models has been widely addressed in the literature, even when $f(\cdot)$ is non monotonic, see [32] and the reference therein. The theoretical identifiability of $f(\cdot)$ is one of the reasons that explain the success of the chemostat model in the mathematical study of the culture of microorganisms.

When two (or more) species $x$ and $y$ are in competition, the model consists simply adding the

\footnotetext{
* Submitted to the editors 2018-11-12.

${ }^{\dagger}$ LAMSIN (LR-99-ES20), ENIT, Université de Tunis El Manar, Tunis, Tunisie and ISIMa, Université de Monastir, Monastir, Tunisie (radhouene.fekihsalem@isima.rnu.tn).

‡ITAP, Irstea, Montpellier SupAgro, Univ Montpellier, Montpellier, France (tewfik.sari@irstea.fr).
} 
consomption terms in the first equation:

$$
\left\{\begin{aligned}
\dot{S} & =D\left(S_{\text {in }}-S\right)-f(S) x-g(S) y \\
\dot{x} & =[f(S)-D] x \\
\dot{y} & =[g(S)-D] y
\end{aligned}\right.
$$

Since equations $f(S)=D$ and $g(S)=D$ cannot have in general a solution, the model (1.2) predicts that at steady state, at most one competitor population avoids extinction. However, the coexistence of competing populations is obvious in nature, and so in order to explain this, it seems necessary to extend the model (1.2). Several mechanisms of coexistence where considered in the literature, among them wall attachment, see [15, 16, 17, 18, 26, 34]. These models were inspired by the Freter model [8,9] of the microflora in the large intestine. Another mechanism that promotes the coexistence is the flocculation of the species, see $[4,5,7,11,12,27,28]$. Attachment and detachment phenomena of bacteria, whether in biofilms on a support [14] or in the form of aggregates or flocs [35] are well known and frequently observed in bacterial growth.

For both previously cited models of wall attachment or flocculation, the total biomass of a given species is decomposed into planktonic (or free) biomass made up of non-attached microorganisms and aggregate (or attached) biomass. Thus, the concentration $x$ of the total biomass is the sum of concentrations $u$ and $v$ of planktonic and aggregate biomass, respectively, $x=u+v$. This distinction permits to take into account different growth and death characteristics according to whether microorganisms are attached or not. Specific velocities $A(\cdot)$ of attachment of free biomass and $B(\cdot)$ of detachment of the attached biomass are introduced in the model. Hence, the general model of flocculation of one species considered in [5] can be written:

$$
\left\{\begin{aligned}
\dot{S} & =D\left(S_{i n}-S\right)-f(S) u-g(S) v \\
\dot{u} & =\left[f(S)-D_{u}\right] u-A(\cdot) u+B(\cdot) v \\
\dot{v} & =\left[g(S)-D_{v}\right] v+A(\cdot) u-B(\cdot) v
\end{aligned}\right.
$$

where $f(S)$ and $g(S)$ represent, respectively, the per-capita growth rates of planktonic and attached bacteria and $D_{u}$ and $D_{v}$ represent, respectively, the disappearance rates of planktonic and attached bacteria. The models of flocculation for several competing species are build as in (1.2), by adding the consomption terms in the dynamic equation of the substrate.

An interesting property of general model (1.3), and its extension to competing species, is that under the assumption that attachment and detachment velocities are fast compared to the specific growth and disappearance rates, using singular perturbation method, see [5, 10, 11, 27], the flocculation model can be reduced to a model with density-dependent growth function. It is well known that density-dependence of the growth functions promotes the coexistence of species $[6,13,21,22,23,24]$. Therefore when attachment and detachment terms are large enough, coexistence is also possible.

The models of attachment or flocculation introduced in the previously cited literature are of the form (1.3), with specific attachment and detachment velocities $A(\cdot)$ and $B(\cdot)$ respectively. For instance, the wall-attachment model of Pilyugin and Waltman [26] corresponds to constant velocities $A(\cdot)=a$ and $B(\cdot)=b$, and the flocculation model of Haegeman and Rapaport [11] corresponds to $A(\cdot)=a u$ and $B(\cdot)=b$, where $a$ and $b$ are constant. For these models, coexistence occurs at positive stable steady state. An extension of the model [11] has been studied in [4, 7] when the growth function of isolated bacteria of the most efficient species presents inhibition. In this case, coexistence can also hold around a stable limit cycle. The reader interested in a review of the various specific attachment and detachment velocities used in the literature is referred to $[5,7]$. See also $[1,17,35]$ for other studies modeling the bacteria flocculation process.

The model in [11] considers flocs of only two bacteria. This model has been extended in [10] to the case of flocs with an arbitrary number of bacteria, by using Smoluchowski's modeling of flocculation [35]. In this modeling, the floc interactions we consider are the aggregation of two flocs to form one bigger floc 
and the breakage of one floc into two smaller ones. Two flocs of sizes $n$ and $m$ (bacteria) respectively, aggregate to form a floc of size $n+m$, with velocity $a_{n, m} u_{n} u_{m}$, proportional to the densities $u_{n}$ and $u_{m}$ of the small flocs. One floc of size $n+m$ (bacteria) splits into two flocs, of sizes $n$ and $m$, respectively, with velocity $b_{n, m} u_{n+m}$, proportional to the density $u_{n+m}$ of the big floc.

In [10], the densities are expressed as the number of flocs per unit of volume. In [11] and in the present paper, $u$ and $v$ are mass densities. Therefore in [11], $u=u_{1} m$ and $v=2 u_{2} m$, where $m$ is the mass of bacteria. Hence, the term $A(\cdot) u=a u^{2}$ in [11] corresponds to the aggregation of two planktonic bacteria to form a floc of size 2 , and the term $B(\cdot) v=b v$ in [11] corresponds to the breakage of one floc into planktonic bacteria. In the present paper, we will not consider the size or the number of bacteria in flocs. We simply distinguish the total biomass $v=\left(2 u_{2}+3 u_{3}+\cdots\right) m$ in flocs and the planktonic biomass $u=u_{1} m$. Therefore, to the term $A(\cdot)=a u$ in [11], we must add a term which is proportional to $v$ and which corresponds to the aggregation of flocs with planktonic bacteria to form bigger flocs. For simplicity, we assume that the coefficient of proportionality is also equal to $a$ and we take $A(\cdot)=a(u+v)$ instead of the more general choice $A(\cdot)=a_{u} u+a_{v} v$. This choice of a linear attachment term was first proposed in [5] and was also considered in [4, 12, 27, 28]. It corresponds to the following flocculation interactions: planktonic bacteria can stick with planktonic bacteria or flocs to form new flocs, with rate $a(u+v) u$, proportional to both the density of isolated bacteria $u$ and the total biomass density $u+v$. Moreover, we assume, as in Smoluchowski's modeling, that $B(\cdot)=b$, that is to say, flocs can split and liberate planktonic bacteria, with rate $b v$, proportional to their density $v$. The model takes the form:

$$
\left\{\begin{aligned}
\dot{S} & =D\left(S_{i n}-S\right)-f(S) u-g(S) v \\
\dot{u} & =\left[f(S)-D_{u}\right] u-a(u+v) u+b v \\
\dot{v} & =\left[g(S)-D_{v}\right] v+a(u+v) u-b v
\end{aligned}\right.
$$

where $a$ and $b$ are positive constants. All these studies restricted to the biologically interesting case $D_{v} \leq D_{u} \leq D$, where $D_{u}=\alpha D$ and $D_{v}=\beta D, \alpha$ and $\beta$ belong to $[0,1]$ and denote, respectively, the fraction of the planktonic and attached bacteria leaving the reactor as proposed by [2] to model a biomass reactor attached to the support or to decouple the residence time of solids and the hydraulic residence time $(1 / D)$. In the present work, we study the model (1.4) where $D_{u}$ and $D_{v}$ can be modeled as in $[25,31]$ by:

$$
D_{u}=\alpha D+m_{u}, \quad D_{v}=\beta D+m_{v}
$$

where the non-negative death (or maintenance) rate parameters $m_{u}$ and $m_{v}$ are taken into consideration.

Indeed, several mathematical studies [3, 19, 29, 30, 36, 37] have attempted to understand the effects of the mortality or the maintenance on the system behavior and the coexistence of species in the chemostat. In $[15,16,17]$, the planktonic cell death rate and adherent cell death rate are considered among the Freter model of biofilm formation by adding an anti-microbial agent to the continuously stirred tank reactor. Thus, a significant death rate of isolated and/or attached bacteria could increase the removal rates $D_{u}$ and/or $D_{v}$ up to values larger than the dilution rate $D$. Therefore the study will not be restricted to the cases $D_{v} \leq D_{u} \leq D$, as in $[4,5,12,27,28]$, and the cases $D<D_{u}, D<D_{v}$ or $D_{u}<D_{v}$, which are also of biological interest, will be investigated.

When $D=D_{u}=D_{v}$, the main result in [12] (see also [27]) was that the model can have a positive steady state $E^{*}=\left(S^{*}, u^{*}, v^{*}\right)$, which exists as long as $f\left(S_{i n}\right)>D$. This steady state is unique and LES as long as it exists. Since $f(S)>g(S)$ (see Hypothesis 2.2 in section 2) the condition $f\left(S_{i n}\right)>D$ of existence of the positive steady state depends only on the growth rate of planktonic bacteria. Therefore the operating diagram which is the bifurcation diagram that illustrates the washout and coexistence regions depends only on the growth rate of planktonic bacteria [28].

It was shown in $[4,5]$ that, if $D_{v}<D_{u} \leq D$, and with monotonic growth rates $f(S)$ and $g(S)$, the model (1.4) can exhibit a bi-stability behavior, similar to the one obtained in (1.1) for non-monotonic kinetics. The case $D_{v}<D_{u} \leq D$ can occur, for instance, if $m_{u}=m_{v}=0$ and $\beta<\alpha$, that is the residence time of the attached bacteria is greater than the one of planktonic bacteria. 
The main objective of this paper is to give a complete analysis of (1.4). We show that when $D_{u} \leq D_{v}$, the model has a positive steady state $E^{*}=\left(S^{*}, u^{*}, v^{*}\right)$, which exists as long as $f\left(S_{i n}\right)>D$. This steady state is unique as long as it exists. If, in addition $D_{v} \leq D, E^{*}$ is LES as long as it exists. Therefore, we extend the results on the existence and uniqueness of the positive steady state obtained in the particular case $D=D_{u}=D_{v}[12,27]$ to the general case $D_{u} \leq D_{v}$, see Proposition 2.6. The result on the stability is extended to the case $D_{u} \leq D_{v} \leq D$, see Proposition 3.5.

Following [4], when $D_{v}<D_{u} \leq D$, we show that multiple positive steady states can appear through saddle-node bifurcations or transcritical bifurcations, see Proposition 3.6. When $D_{v}<D_{u}$ and $D<D_{u}$, we investigate numerically the system and we see the occurrence of Hopf bifurcations and homoclinic bifurcations, see subsections 4.1 and 4.2. Notice that the condition $D<D_{u}$ or $D<D_{v}$ can occur only when mortality (or maintenance) terms are added to the model $\left(D_{u}=D+m_{u}\right)$. Therefore the destabilization of the positive steady state is due to the mortality of the species, and is similar to some results obtained in the existing literature on food webs (predator-prey model) in the chemostat where the addition of mortality terms of the species lead to destabilization of the system $[3,19]$.

The paper is organized as follows. The next section presents general assumptions for the growth functions of flocculation model (1.4) and the analysis of the existence of steady states. In section 3, the asymptotic behavior analysis of model (1.4) was done according to the dilution rate and the disappearance rates of planktonic and attached bacteria. Considering growth rates of Monod-type, numerical simulations are presented in section 4 in order to show the emergence of limit cycles and the multiplicity of positive steady states when the system exhibits the bi-stability. Finally, conclusions are drawn in the last section 5 . The proofs of some propositions and technical lemmas are reported in Appendix A.

2. Assumptions on the model and steady states. We use the following general assumptions for the growth functions $f(S)$ and $g(S)$ :

Hypothesis 2.1. $f(0)=g(0)=0$ and $f^{\prime}(S)>0$ and $g^{\prime}(S)>0$ for all $S>0$.

Hypothesis 2.2. $f(S)>g(S)$ for all $S>0$.

Hypothesis 2.1 means that the growth can take place if and only if the substrate is present. In addition, the growth rates of isolated and attached bacteria increase with the concentration of substrate. Hypothesis 2.2 means that bacteria in flocs consume less substrate than isolated bacteria, this means that a lower specific growth rate. In fact, the flocs consume less substrate than isolated bacteria since they have less access to substrate, given that this access to substrate is proportional to the outside surface of flocs.

In order to preserve the biological significance of our model (1.4), we will show that solutions of ordinary differential equations are non-negative and bounded for any non-negative time.

Proposition 2.3. For any non-negative initial condition, the solutions of system (1.4) remain nonnegative and positively bounded. In addition, the set

$$
\Omega=\left\{(S, u, v) \in \mathbb{R}_{+}^{3}: S+u+v \leq \frac{D}{D_{\min }} S_{i n}\right\}, \quad \text { where } \quad D_{\min }=\min \left(D, D_{u}, D_{v}\right),
$$

is positively invariant and is a global attractor for the dynamics (1.4).

The proof is given in Appendix A.1.

The first step is to determine the steady states of (1.4). A steady state $\left(S^{*}, u^{*}, v^{*}\right)$ must be a solution of the system

$$
\left\{\begin{array}{l}
0=D\left(S_{i n}-S^{*}\right)-f\left(S^{*}\right) u^{*}-g\left(S^{*}\right) v^{*} \\
0=\left[f\left(S^{*}\right)-D_{u}\right] u^{*}-a\left(u^{*}+v^{*}\right) u^{*}+b v^{*} \\
0=\left[g\left(S^{*}\right)-D_{v}\right] v^{*}+a\left(u^{*}+v^{*}\right) u^{*}-b v^{*} .
\end{array}\right.
$$

To solve (2.1), we use a method similar to the concept of steady-state characteristic that is introduced by Lobry et al. $[23,24]$. This concept consists of determining the steady states of the second and third 
equations of (1.4), where $S^{*}$ is considered as an input of the system. This means that we must solve the second and third equation of (2.1), where $u^{*}$ and $v^{*}$ are unknown and $S^{*}$ is considered as a parameter. Assume that one obtains

$$
u^{*}=U\left(S^{*}\right), \quad v^{*}=V\left(S^{*}\right) .
$$

If we replace $u^{*}$ and $v^{*}$ by these expressions in the first equation of (2.1), we obtain an equation in the sole variable $S^{*}$ of the form

$$
D\left(S_{\text {in }}-S^{*}\right)=H\left(S^{*}\right), \quad \text { where } \quad H\left(S^{*}\right)=f\left(S^{*}\right) U\left(S^{*}\right)+g\left(S^{*}\right) V\left(S^{*}\right) .
$$

Solving this equation, we find $S^{*}$ and hence $u^{*}=U\left(S^{*}\right)$ and $v^{*}=V\left(S^{*}\right)$. In the sequel, we show how to determine the functions $U, V$ and $H$ and we give the conditions such that a solution $S^{*}$ exists.

From Hypothesis 2.1, when equations $f(S)=D_{u}, g(S)=D_{v}$ and $g(S)=D_{v}+b$ have solutions, they are unique and we define the usual break-even concentrations

$$
\lambda_{u}=f^{-1}\left(D_{u}\right), \quad \lambda_{v}=g^{-1}\left(D_{v}\right) \quad \text { and } \quad \lambda_{b}=g^{-1}\left(D_{v}+b\right) .
$$

From Hypothesis 2.2, if in addition $D_{v} \geq D_{u}$, then $\lambda_{v}>\lambda_{u}$. When equations $f(S)=D_{u}$ or $g(S)=D_{v}$ or $g(S)=D_{v}+b$ have no solution, we put $\lambda_{u}=\infty$ or $\lambda_{v}=\infty$ or $\lambda_{b}=\infty$. We define the interval $I$ by (see Figure 1):

$$
I=\left\{\begin{array}{lll}
] \lambda_{u}, \lambda_{v}[ & \text { if } & \lambda_{u}<\lambda_{v} \\
] \lambda_{v}, \min \left(\lambda_{u}, \lambda_{b}\right)[ & \text { if } & \lambda_{u}>\lambda_{v}
\end{array}\right.
$$

Notice that $\varphi\left(\lambda_{u}\right)=\psi\left(\lambda_{v}\right)=0$ and $\psi\left(\lambda_{b}\right)=b$, where functions $\varphi$ and $\psi$ are defined by:

$$
\varphi(S)=f(S)-D_{u} \quad \text { and } \quad \psi(S)=g(S)-D_{v} .
$$
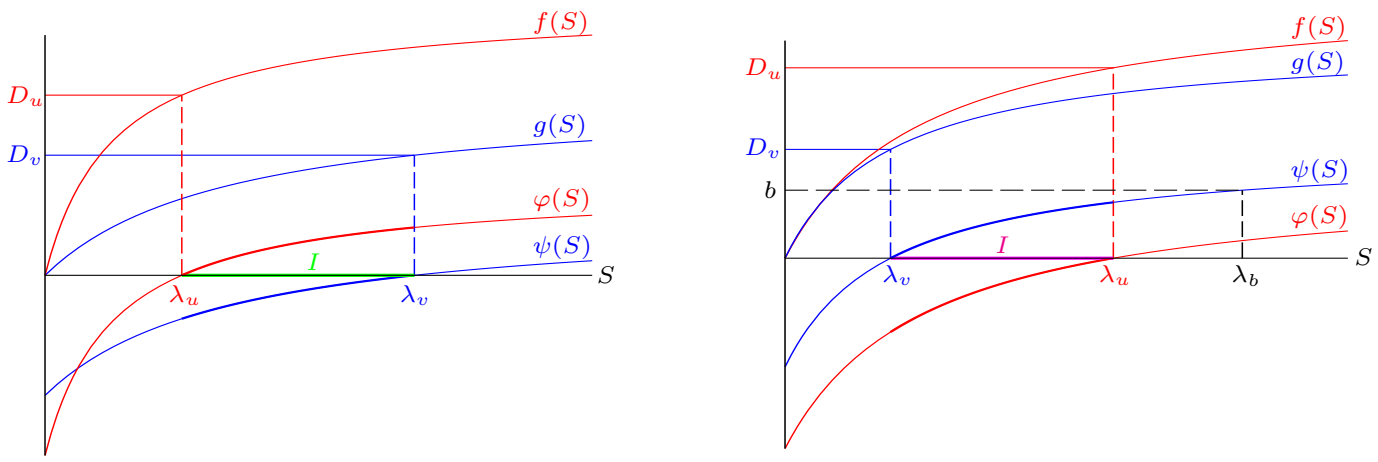

FIG. 1. Definition of the interval I: (a) the case $\lambda_{u}<\lambda_{v}$; (b) the case $\lambda_{v}<\min \left(\lambda_{u}, \lambda_{b}\right)$.

In the rest of the paper, we use also the following notations:

$$
\begin{gathered}
U(S):=\frac{\varphi(S)(\psi(S)-b)}{a[\psi(S)-\varphi(S)]} \text { and } V(S):=-\frac{\varphi^{2}(S)(\psi(S)-b)}{a[\psi(S)-\varphi(S)] \psi(S)}, \\
H(S):=f(S) U(S)+g(S) V(S) .
\end{gathered}
$$

We can state the following result:

Lemma 2.4. Assume that Hypotheses 2.1 and 2.2 hold. Then system (1.4) has the following steady states: 
1. the washout $E_{0}=\left(S_{i n}, 0,0\right)$, that always exists,

2. a positive steady state, $E_{1}=\left(S^{*}, u^{*}, v^{*}\right)$ with $S^{*}$ solution

$$
D\left(S_{\text {in }}-S^{*}\right)=H\left(S^{*}\right)
$$

where $H$ is given by (2.5) and $u^{*}=U\left(S^{*}\right)$ and $v^{*}=V\left(S^{*}\right)$, where $U$ and $V$ are given by (2.4). This positive steady state exists if and only if $S^{*} \in I$ where $I$ is defined by (2.2).

Proof. From the second equation of (2.1), if $u^{*}=0$, it follows that $v^{*}=0$. From the last equation of $(2.1)$, if $v^{*}=0$, then $u^{*}=0$. Hence, we cannot have a steady state of extinction only of planktonic or attached bacteria. Therefore, besides the washout steady state

$$
E_{0}=\left(S_{i n}, 0,0\right)
$$

where both planktonic and attached bacteria are extinct, the system can have a positive steady state of coexistence

$$
E_{1}=\left(S^{*}, u^{*}, v^{*}\right)
$$

where $S^{*}>0, u^{*}>0$ and $v^{*}>0$. Making the sum of the second and the third equation of (2.1), we obtain

$$
\varphi\left(S^{*}\right) u^{*}+\psi\left(S^{*}\right) v^{*}=0,
$$

where $\varphi$ and $\psi$ are given by (2.3). This equation admits positive solutions $u^{*}$ and $v^{*}$ if and only if $\varphi\left(S^{*}\right)$ and $\psi\left(S^{*}\right)$ have opposite signs, i.e. $S^{*}$ is between $\lambda_{u}$ and $\lambda_{v}$, see Figure 1. In this case, $\psi\left(S^{*}\right) \neq 0$ and equation (2.7) can be rewritten as

$$
v^{*}=-\frac{\varphi\left(S^{*}\right)}{\psi\left(S^{*}\right)} u^{*} .
$$

Replacing $v^{*}$ by its expression (2.8) in the second equation of (2.1), we obtain

$$
u^{*}=U\left(S^{*}\right),
$$

where $U$ is given by (2.4). Note that $u^{*}$ defined by (2.9) is positive if and only if $\lambda_{u}<S^{*}<\lambda_{v}$ or $\lambda_{v}<S^{*}<\min \left(\lambda_{b}, \lambda_{u}\right)$, that is to say $S^{*} \in I$. Therefore we must seek the solutions $\left(S^{*}, u^{*}, v^{*}\right)$ of $(2.1)$ such that $S^{*} \in I$. By replacing $u^{*}$ by (2.9) in (2.8), we obtain

$$
v^{*}=V\left(S^{*}\right),
$$

where $V$ is given by (2.4). Making the sum of three equations of (2.1) and replacing $u^{*}$ and $v^{*}$ by (2.9) and (2.10), we obtain that $S^{*}$ is a solution of (2.6).

Each solution of equation (2.6) belonging to the interval $I$ give rise to a positive steady state of the system. Straightforward calculation yields

$$
H^{\prime}(S)=\frac{f^{\prime}(\psi-b) \psi F_{0}+g^{\prime} \varphi G_{0}}{a(\psi-\varphi)^{2} \psi^{2}}
$$

where

$$
F_{0}(S)=D_{u} \psi^{2}-2 D_{v} \varphi \psi+D_{v} \varphi^{2} \quad \text { and } \quad G_{0}(S)=b D_{u} \psi^{2}+\left(D_{v}-D_{u}\right) \varphi \psi^{2}+b D_{v}\left(\varphi^{2}-2 \varphi \psi\right) .
$$

We have the following technical lemma:

Lemma 2.5. If $D_{u} \leq D_{v}$, then $\lambda_{u}<\lambda_{v}$ and $H^{\prime}(S)>0$ on $I$. If $D_{u}>D_{v}$, then the following two cases must be distinguished: 
- Case $\lambda_{u}<\lambda_{v}$ : the sign of $H^{\prime}(S)$ can be positive or negative for $S \in I$.

- Case $\lambda_{u}>\lambda_{v}$ : one has $H^{\prime}(S)<0$ on $I$.

Proof. Recall that $\varphi$ and $\psi$ have opposite signs on $I$ and then $F_{0}(S)>0$ for all $S \in I$. From Hypothesis 2.2, if $D_{u} \leq D_{v}$, then $\lambda_{u}<\lambda_{v}$. Therefore, $\varphi>0$ and $\psi<0$ on $I$. From (2.11) and (2.12), it follows that $H^{\prime}(S)>0$ on $I$. Let $D_{u}>D_{v}$. If $\lambda_{u}<\lambda_{v}$, then $\varphi>0$ and $\psi<0$ on $I$. Hence, the sign of $G_{0}(S)$ can be positive or negative at $S \in I$ so that $H^{\prime}(S)$ can change sign at $S \in I$. If $\lambda_{u}>\lambda_{v}$, then $\varphi<0$ and $0<\psi<b$ on $I$. Hence, $G_{0}(S)>0$ on $I$ and as $F_{0}(S)>0$ on $I$, it follows that $H^{\prime}(S)<0$ on $I$.

The following proposition exhibits the number of positive steady states of (1.4).

Proposition 2.6.

- When $D_{u} \leq D_{v}$, then the positive steady state $E_{1}=\left(S^{*}, u^{*}, v^{*}\right)$ exists if and only if $S_{i n}>\lambda_{u}$. If it exists, it is unique.

- When $D_{u}>D_{v}$, then there exists at least one positive steady state in the case $\lambda_{u}<\min \left(\lambda_{v}, S_{i n}\right)$ or $\lambda_{v}<\min \left(\lambda_{u}, \lambda_{b}\right)<S_{i n}$. Generically, the system can have generically an odd number of positive steady states. When $S_{i n}<\min \left(\lambda_{u}, \lambda_{b}\right)$ and $\lambda_{v}<\lambda_{u}$, then generically the system has no positive steady state or an even number of positive steady states.

Proof. The positive steady states are given by the intersection of the line $\delta$ of equation $y=D\left(S_{i n}-S\right)$ and the curve of function $H(\cdot)$.

In the case where $D_{u} \leq D_{v}$, it follows from Lemma 2.5 that $\lambda_{u}<\lambda_{v}$. In this case, the function $H(\cdot)$ is defined and positive on the interval $I=] \lambda_{u}, \lambda_{v}[$ since $\varphi(S)>0$ and $\psi(S)<0$ (see Figure 13(a)). Moreover, it vanishes at $\lambda_{u}$ and tends to infinity as $S$ tends to $\lambda_{v}$. Thus, equation (2.6) has a solution $S^{*} \in I$ if and only if $S_{i n}>\lambda_{u}$ (see Figure 13(a)). In addition, the function $H(\cdot)$ is increasing and then $E_{1}$ is unique if it exists.

In the case where $D_{u}>D_{v}$, it follows from Lemma 2.5 that equation (2.6) may have several solutions whose number is generically odd in the case $\lambda_{u}<\min \left(\lambda_{v}, S_{i n}\right)$ or $\lambda_{v}<\min \left(\lambda_{u}, \lambda_{b}\right)<S_{\text {in }}$ (see Figures 13, 14, 16, and 17(a)) and even in the case $\lambda_{v}<S_{i n}<\min \left(\lambda_{u}, \lambda_{b}\right)$ (see Figures 15 and 18(a)). Indeed, in the case $\lambda_{u}>\lambda_{v}$, the function $H(\cdot)$ is defined and positive on the interval $I$ since $\varphi(S)<0$ and $0<\psi(S)<b$.

When model (1.4) can have multiple positive steady states, the following results show that the positive steady state that has less substrate can promote the planktonic and/or aggregated biomass according to break-even concentrations $\lambda_{u}$ and $\lambda_{v}$.

Proposition 2.7. Let $E_{1}=\left(S^{*}, u^{*}, v^{*}\right)$ and $E_{2}=\left(S^{* *}, u^{* *}, v^{* *}\right)$ be two positive steady states of (1.4) such that $S^{*}<S^{* *}$.

1. If $\lambda_{u}<\lambda_{v}$, then $u^{*}>u^{* *}$ and $v^{*}<v^{* *}$.

2. If $\lambda_{u}>\lambda_{v}$, then $u^{*}>u^{* *}$ and $v^{*}>v^{* *}$.

The proof is given in Appendix A.1.

3. Stability of steady states. In this section, we focus on the study of local asymptotic stability of each steady state of system (1.4). Let $J$ be the Jacobian matrix of (1.4) at $(S, u, v)$, that is given by

$$
J=\left[\begin{array}{ccc}
-D-f^{\prime}(S) u-g^{\prime}(S) v & -f(S) & -g(S) \\
f^{\prime}(S) u & \varphi(S)-a(2 u+v) & -a u+b \\
g^{\prime}(S) v & a(2 u+v) & \psi(S)+a u-b
\end{array}\right]
$$

The stability of the washout steady state is given as follows:

Proposition 3.1. $E_{0}$ is LES if and only if $S_{i n}<\lambda_{u}$ and $S_{i n}<\lambda_{b}$. 
Proof. At $E_{0}=\left(S_{i n}, 0,0\right)$, the Jacobian matrix (3.1) is written as follows:

$$
J_{0}=\left[\begin{array}{ccc}
-D & -f\left(S_{i n}\right) & -g\left(S_{i n}\right) \\
0 & \varphi\left(S_{i n}\right) & b \\
0 & 0 & \psi\left(S_{i n}\right)-b
\end{array}\right]
$$

The eigenvalues are $-D, \varphi\left(S_{i n}\right)$ and $\psi\left(S_{i n}\right)-b$ which are negative if and only if $S_{i n}<\lambda_{u}$ and $S_{i n}<\lambda_{b}$. $\square$ In what follows, we analyze the stability of positive steady states. The Jacobian matrix at $E_{1}=$ $\left(S^{*}, u^{*}, v^{*}\right)$ is given by

$$
J_{1}=\left[\begin{array}{ccc}
-m_{11} & -m_{12} & -m_{13} \\
m_{21} & -m_{22} & a_{23} \\
m_{31} & m_{32} & -m_{33}
\end{array}\right]
$$

where

$$
\left\{\begin{array}{l}
m_{11}=D+f^{\prime}\left(S^{*}\right) u^{*}+g^{\prime}\left(S^{*}\right) v^{*}, \quad m_{12}=f\left(S^{*}\right), \quad m_{13}=g\left(S^{*}\right), \\
m_{21}=f^{\prime}\left(S^{*}\right) u^{*}, \quad m_{22}=a\left(2 u^{*}+v^{*}\right)-\varphi\left(S^{*}\right), \quad a_{23}=b-a u^{*}, \\
m_{31}=g^{\prime}\left(S^{*}\right) v^{*}, \quad m_{32}=a\left(2 u^{*}+v^{*}\right) \quad \text { and } \quad m_{33}=b-a u^{*}-\psi\left(S^{*}\right) .
\end{array}\right.
$$

The characteristic polynomial is given by

$$
P(\lambda)=\lambda^{3}+c_{1} \lambda^{2}+c_{2} \lambda+c_{3},
$$

where

$$
\begin{gathered}
c_{1}=m_{11}+m_{22}+m_{33}, \\
c_{2}=m_{12} m_{21}+m_{13} m_{31}-m_{32} a_{23}+m_{11} m_{22}+m_{11} m_{33}+m_{22} m_{33}, \\
c_{3}=m_{11}\left(m_{22} m_{33}-m_{32} a_{23}\right)+m_{21}\left(m_{12} m_{33}+m_{32} m_{13}\right)+m_{31}\left(m_{12} a_{23}+m_{13} m_{22}\right) .
\end{gathered}
$$

According to the Routh-Hurwitz criterion, $E_{1}$ is LES if and only if

$$
c_{1}>0, \quad c_{3}>0 \text { and } c_{4}=c_{1} c_{2}-c_{3}>0 .
$$

We have the following result:

Lemma 3.2. All $m_{i j}$ are positive for all $i, j=1, \ldots, 3$ with $(i, j) \neq(2,3)$ and we have $c_{1}>0$.

The proof is given in Appendix A.2.

In the next lemma, we will show that the sign of $c_{3}$ is given by the position of the curve of function $H(\cdot)$ with respect to the line of equation $y=D\left(S_{i n}-S\right)$. More precisely, we give the link between the determinant of the Jacobian matrix $J_{1}$ at $E_{1}=\left(S^{*}, u^{*}, v^{*}\right)$ and $D+H^{\prime}\left(S^{*}\right)$. Indeed, this result is very general, as we show in Appendix A.3.

Proposition 3.3. One has $c_{3}=-\operatorname{det}\left(J_{1}\right)=-\varphi\left(S^{*}\right)\left(\psi\left(S^{*}\right)-b\right)\left(D+H^{\prime}\left(S^{*}\right)\right)$.

The proof is given in Appendix A.3.

Since the condition $c_{4}>0$ given by (A.10) of the Routh-Hurwitz criterion (3.4) could be unfulfilled, we will study the behavior of flocculation model (1.4) according to the dilution rate and the disappearance rates of planktonic and attached bacteria. In fact, there exist four cases that must be distinguished (see Figure 2):

$$
\begin{array}{llll}
\text { Case 1: } & D_{u} \leq D_{v} \leq D, & \text { Case 2: } & D_{v}<D_{u} \leq D, \\
\text { Case 3: } & D_{v}<D_{u} \text { and } D<D_{u}, & \text { Case 4: } & D_{u} \leq D_{v} \text { and } D<D_{v} .
\end{array}
$$

To determine the local stability of the positive steady state in the first and second cases of (3.5), we will have need of the following. 


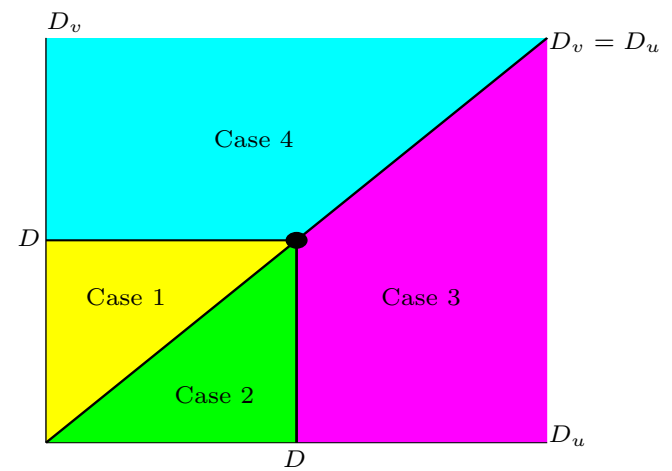

Fig. 2. Divers regions according to $D, D_{u}$ and $D_{v}$ when the behavior of system (1.4) is different. Yellow region for case $1\left(D_{u} \leq D_{v} \leq D\right)$; green region for case $2\left(D_{v}<D_{u} \leq D\right)$; magenta region for case $3\left(D_{v}<D_{u}\right.$ and $\left.D<D_{u}\right)$; cyan region for case $4\left(D_{u} \leq D_{v}\right.$ and $\left.D<D_{v}\right)$.

Proposition 3.4. In the cases 1 and $2\left(D_{u} \leq D\right.$ and $\left.D_{v} \leq D\right)$, we have $c_{4}>0$.

The proof is given in Appendix A.4.

It was shown in [12], see also $[27,28]$ that if $D_{u}=D_{v}=D$ (which is represented by a point in Figure 2) then the positive steady state $E_{1}$ exists and is unique and LES if and only if $S_{i n}>\lambda_{u}$. Actually, this result holds in case 1.

Proposition 3.5. In the case $1\left(D_{u} \leq D_{v} \leq D\right)$, the positive steady state $E_{1}=\left(S^{*}, u^{*}, v^{*}\right)$ exists if and only if $S_{i n}>\lambda_{u}$. If it exists, it is unique and LES.

Proof. From Proposition 2.6, $E_{1}$ exists if and only if $S_{i n}>\lambda_{u}$. If it exists, it is unique. From Lemma 2.5, one has $\lambda_{u}<\lambda_{v}$ and $H^{\prime}(S)>0$ on $I$. From Proposition 3.3 and Proposition 3.4, it follows that $E_{1}$ is LES as soon as it exists.

The case 2 was solved in [4] where it was shown that the stability depends only on the relative position of the curve of function $y=H(S)$ and the straight line of equation $y=D\left(S_{i n}-S\right)$ that is to say, on the sign of $D+H^{\prime}\left(S^{*}\right)$, as shown in Figure 3. More precisely, we have:

Proposition 3.6. Let $E_{1}=\left(S^{*}, u^{*}, v^{*}\right)$ be a positive steady state. Assume that case 2 holds.

1. If $\lambda_{u}<\lambda_{v}$ : $E_{1}$ is LES if $H^{\prime}\left(S^{*}\right)>-D$ and is unstable if $H^{\prime}\left(S^{*}\right)<-D$.

2. If $\lambda_{u}>\lambda_{v}: E_{1}$ is LES if $H^{\prime}\left(S^{*}\right)<-D$ and is unstable if $H^{\prime}\left(S^{*}\right)>-D$.

Proof. According to Lemma 3.2 and Proposition 3.4, we have $c_{1}>0$ and $c_{4}>0$. Therefore, the positive steady state is LES if and only if the remaining condition $c_{3}>0$ in the Routh-Hurwitz criterion (3.4) is satisfied.

In the case $\lambda_{u}<\lambda_{v}$, we have $\varphi\left(S^{*}\right)>0$ and $\psi\left(S^{*}\right)<0$. From Proposition 3.3, if $H^{\prime}\left(S^{*}\right)<-D$, it follows that $c_{3}<0$. Therefore, the positive steady state is unstable. If $H^{\prime}\left(S^{*}\right)>-D$, it follows that $c_{3}>0$ and hence the positive steady state is LES.

In the case $\lambda_{u}>\lambda_{v}$, we have $\varphi\left(S^{*}\right)<0$ and $0<\psi\left(S^{*}\right)<b$. From Proposition 3.3, if $H^{\prime}\left(S^{*}\right)>-D$, it follows that $c_{3}<0$. Therefore, the positive steady state is unstable. If $H^{\prime}\left(S^{*}\right)<-D$, it follows that $c_{3}>0$ and hence the positive steady state is LES.

In the case 3 of (3.5), we will show that $c_{4}$ can change sign by varying the control parameter $S_{i n}$ such that the positive steady state $E_{1}$ could change its behavior without any collision with another steady state. Indeed, we succeeded in finding a set of parameters where $E_{1}$ change stability through a Hopf bifurcation [20], as we shall see in the next section. More precisely, we show numerically the occurrence of limit cycles in the case 3 of (3.5) when

$$
D<D_{v} \leq D_{u} \quad \text { or } \quad D_{v}<D \leq D_{u} .
$$

In the case 4 of (3.5) we always have $\lambda_{u}<\lambda_{v}$ and $H^{\prime}(S)>0$, as shown in Lemma 2.5. Therefore, from Proposition 3.3, it is deduced that in the case 4 of (3.5) we always have $c_{3}>0$. We were not able to find 

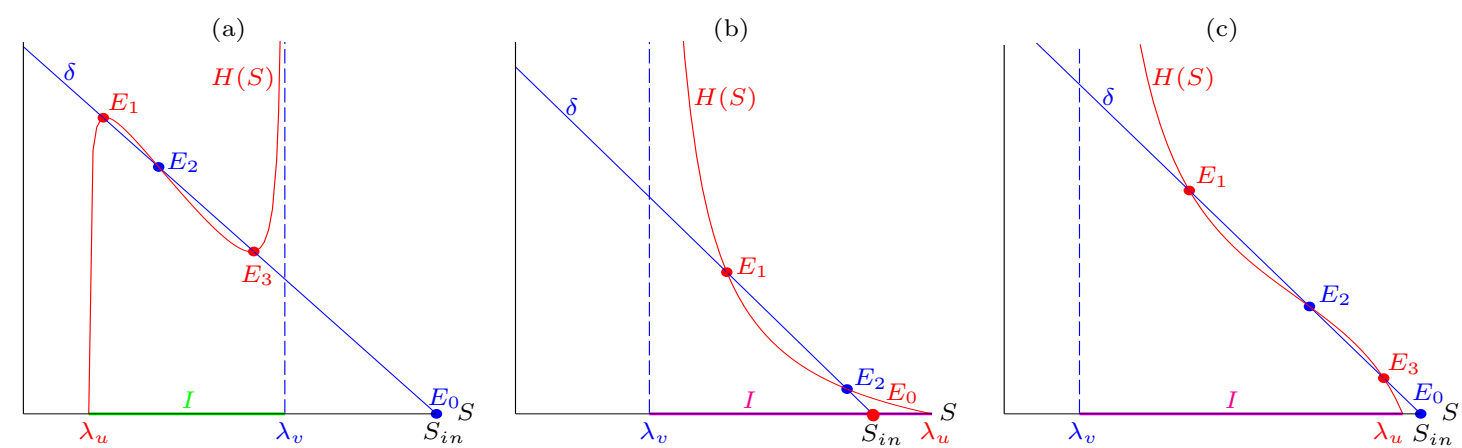

FIG. 3. Existence and stability of steady states in the second case of (3.5): (a) when $\lambda_{u}<\min \left(\lambda_{v}, S_{i n}\right)$, (b) when $\lambda_{v}<S_{i n}<\lambda_{u}<\lambda_{b}$ and (c) when $S_{i n}>\min \left(\lambda_{u}, \lambda_{b}\right)$. In all figures, we have chosen the red color for LES steady states and blue color for unstable steady states.

a set of parameters for which $c_{4}<0$, as in the case 3 of (3.5) and we conjecture that in this case the positive steady state $E_{1}$ which is unique as soon as it exists, is also LES as soon as it exists.

In Figure 2, yellow (case 1) and cyan (case 4) colors represent the region where the system has at most one positive steady state with $c_{3}>0$. Green (case 2) and magenta (case 3) colors represent the region where the system can have a multiplicity of positive steady state where the sign of $c_{3}$ can be positive or negative. In yellow and green regions, $c_{4}$ is positive. In magenta region, we can have $c_{3}>0$ and $c_{4}<0$. In cyan region, we conjecture that $c_{4}>0$.

\section{Numerical simulations.}

4.1. Occurrence of limit cycle: case 3 when $D<D_{v}<D_{u}$. In order to show that the condition $c_{4}\left(S^{*}\right)>0$ evaluated at $E_{1}=\left(S^{*}, u^{*}, v^{*}\right)$ could be unfulfilled and to detect if the positive steady state $E_{1}$ can change stability through a Hopf bifurcation, all biological parameters were fixed such that $D<D_{v}<D_{u}$. Then, the control parameter $S_{i n}$ was varied.

To see the change of sign of the function $S^{*} \mapsto c_{4}\left(S^{*}\right)$ evaluated at $E_{1}$ and to detect the occurrence of limit cycles, it is useful to illustrate the curve of this function. To this end, the growth rates $f$ and $g$ are chosen for simplicity of Monod-type

$$
f(S)=\frac{m_{1} S}{k_{1}+S} \quad \text { and } \quad g(S)=\frac{m_{2} S}{k_{2}+S}
$$

where $m_{i}$ denotes the maximum growth rate and $k_{i}$ the half-saturation constant. Indeed, we succeeded in finding a set of parameters such that $c_{4}$ can change its sign as $S_{i n}$ increases (or equivalently $S^{*}$ decreases) (see Figure 4). The parameter values used for the simulations are provided in Table 1.

(a)

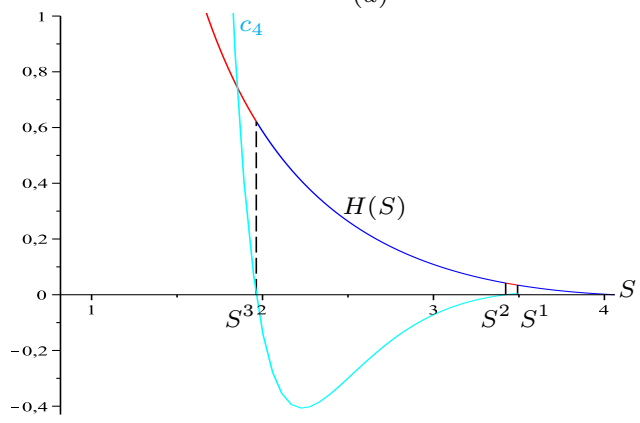

$(b)$

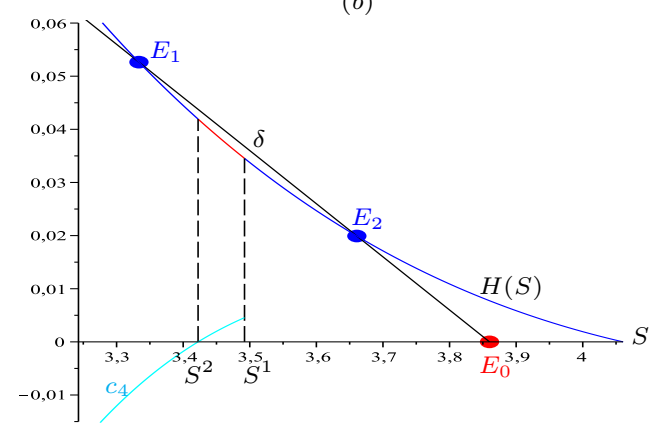

FIG. 4. (a) Change of sign of $c_{4}$ and the corresponding stability of $E_{1}$ on the red or blue curve of function $H(\cdot)$ where $S^{1} \approx 3.492$ (or equivalently $S_{\text {in }}^{1} \approx 3.837$ ), $S^{2} \approx 3.422$ (or $S_{\text {in }}^{2} \approx 3.842$ ) and $S^{3} \approx 1.963$ (or $S_{\text {in }}^{3} \approx 8.179$ ), (b) a magnification for $3.25<S<\lambda_{u}=4.061$ where $S_{i n}=3.86$. 
The solution $S^{1}$ of equation $H^{\prime}(S)=-D$ and solutions $S^{i}, i=2,3$, of equation $c_{4}(S)=0$ are represented in Figure 4. In fact, $S_{i n}^{1}$ is a critical value of $S_{i n}$ for which the curve of function $H(\cdot)$ is tangent to the line $\delta$ of equation $y=D\left(S_{i n}-S\right)$. In addition, $S_{i n}^{i}, i=1,2,3$ can be computed explicitly by

$$
S_{i n}^{i}=\frac{1}{D} H\left(S^{i}\right)+S^{i} .
$$

According to control parameter $S_{i n}$, the following changes of stability of $E_{0}$ and $E_{1}$ occur when the steady state $E_{2}$ is unstable whenever it exists.

- For $S_{i n} \in\left[0, S_{i n}^{1}\left[\right.\right.$, there exists a unique steady state which is the washout $E_{0}$ and it is LES.

- For $S_{i n}=S_{i n}^{1}$, two positive steady states $E_{1}$ and $E_{2}$ bifurcate into the positive quadrant through a saddle-node bifurcation.

- For $\left.S_{i n} \in\right] S_{i n}^{1}, S_{i n}^{2}\left[\right.$, (or equivalently $\left.S^{*} \in\right] S^{2}, S^{1}\left[\right.$ ), $c_{4}\left(S^{*}\right)>0$ and $H^{\prime}\left(S^{*}\right)<-D$ (see Figure 4(b)). It follows that $E_{2}$ is unstable while $E_{0}$ and $E_{1}$ are LES.

- For $\left.S_{i n} \in\right] S_{i n}^{2}, \lambda_{u}\left[, E_{0}\right.$ is LES while $E_{1}$ and $E_{2}$ are unstable where $c_{4}<0$ (see Figure 4(b)).

- For $S_{i n}=\lambda_{u}, E_{2}$ coalesces with $E_{0}$.

- For $\left.S_{i n} \in\right] \lambda_{u}, S_{i n}^{3}\left[, E_{2}\right.$ disappears through a transcritical bifurcation and transfers instability to $E_{0}$ while $E_{1}$ still unstable.

- For $\left.S_{i n} \in\right] S_{i n}^{3},+\infty\left[\right.$, (or equivalently $\left.S^{*}<S^{3}\right), c_{4}\left(S^{*}\right)>0$ and $H^{\prime}\left(S^{*}\right)<-D$ (see Figure 4(a)). It follows that $E_{0}$ is unstable and $E_{1}$ changes its stability and becomes LES.

To understand and analyze these changes of local behavior of $E_{1}$ in $S_{i n}^{2}$ and $S_{i n}^{3}$ without any bifurcation with other steady states, we determine numerically the eigenvalues of the Jacobian matrix $J_{1}$ of system (1.4) at the positive steady state $E_{1}$.

$(a)$

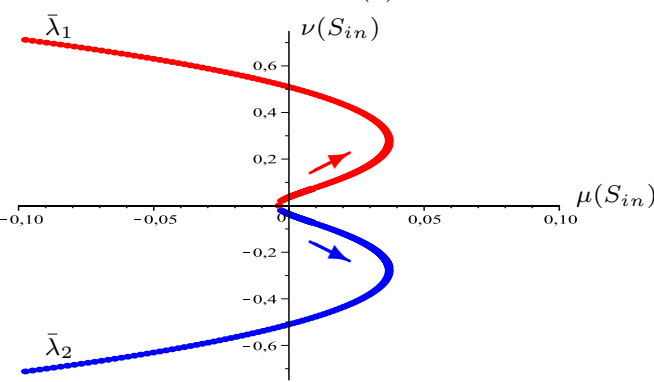

(b)

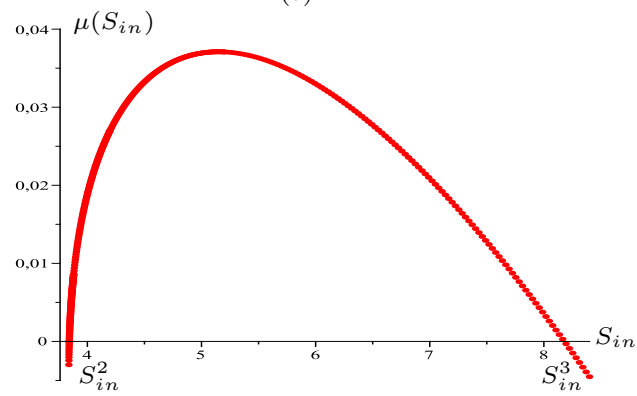

FIG. 5. Two supercritical Hopf bifurcations: (a) variation of a pair of complex-conjugate eigenvalues as $S_{\text {in }}$ increases and the corresponding real part (b) as a function of $S_{i n}$.

Indeed, the Jacobian matrix $J_{1}$ of system (1.4) at $E_{1}$ has one negative eigenvalue and one pair of complex-conjugate eigenvalues

$$
\lambda_{j}\left(S_{i n}\right)=\mu\left(S_{i n}\right) \pm i \nu\left(S_{i n}\right), \quad j=1,2 .
$$

Increasing the control parameter $S_{i n}$ from $S_{i n}^{1}$, this pair crosses the imaginary axis at the critical value $S_{i n}=S_{i n}^{2}$ from negative half plane to positive half plane (see Figure 5(a)), that is, it becomes purely imaginary for $S_{i n}^{2}$ such that $\mu\left(S_{i n}^{2}\right)=0$, with $\nu\left(S_{i n}^{2}\right) \neq 0$. The following inequality is checked numerically

$$
\frac{d \mu}{d S_{i n}}\left(S_{i n}^{2}\right)>0 \text {. }
$$

Thus, $E_{1}$ is obviously stable focus node on the red curve for $\left.S_{i n} \in\right] S_{i n}^{1}, S_{i n}^{2}$ [ but becomes saddle focus on the blue curve for $\left.S_{i n} \in\right] S_{i n}^{2}, S_{i n}^{3}$ [ (see Figure 4(b)) undergoing a supercritical Hopf bifurcation at $S_{i n}=S_{i n}^{2}$, with orbits spiralling out (see Figure 6). Indeed, the bifurcation is supercritical since a unique stable limit cycle bifurcates from the steady state $E_{1}$ for $S_{i n}=S_{i n}^{2}$.

Increasing $S_{i n}$ further, this pair enters to the positive half plane and then returns to the negative half plane by crossing again the imaginary axis at $S_{i n}=S_{i n}^{3}$ (see Figure $5\left(\right.$ a)). Similarly, $E_{1}$ changes again 
their stability and returns stable focus node on the red curve for $S_{i n}>S_{i n}^{3}$ (see Figure 4(a)) due to the supercritical Hopf bifurcation at $S_{i n}=S_{i n}^{3}$. Figure 5(b) shows these critical values and the real part of the complex-conjugate eigenvalues, as a function of $S_{i n}$.
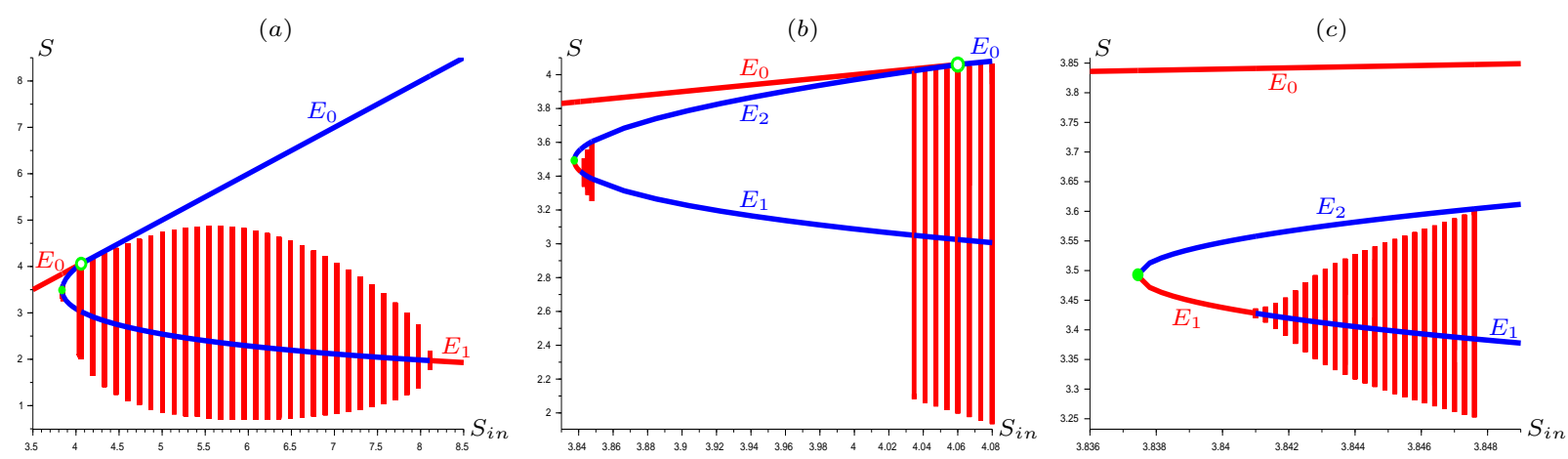

FIG. 6. (a) Scilab simulation showing projections of the $\omega$-limit set in variable $S$ when $S_{i n} \in[3.5,8.5]$ reveals the emergence and the disappearance of limit cycles; (b) a magnification of two homoclinic bifurcations when $S_{i n} \in[3.83,4.08]$; (c) a magnification of supercritical Hopf bifurcation when $S_{i n} \in[3.835,3.85]$. A green dot and a green open circle represent a saddle-node bifurcation and transcritical bifurcation, respectively.

In order to illustrate the occurrence of limit cycle and to understand what happens with the limit cycle born via the supercritical Hopf bifurcation when $S_{i n}$ varied, we represent in Figure 6 the oneparameter bifurcation diagram for system (1.4) when all other parameters are fixed. The $\omega$-limit set projected in coordinate $S$ depending on the control parameter $S_{i n}$ shows that the "small" limit cycles born at $S_{i n}^{2}$ (see Figure 6(b)). When magnified, we observe more clearly the occurrence of limit cycle and then the disappearance via orbits homoclinic to the saddle point $E_{2}$ at $S_{i n}=S_{i n}^{h_{1}}=3.8477$ (see Figure 6(c)). When $S_{i n}$ decreases, the stable limit cycle which appears via supercritical Hopf bifurcation at $S_{i n}=S_{i n}^{3}=8.179$, will disappear via orbits homoclinic to the saddle point $E_{2}$ at $S_{i n}=S_{i n}^{h_{2}}=4.03468$.

In order to show the behavior of system according to initial conditions, we illustrate in the following the time course and the three-dimensional phase plot in most important cases.

(a)

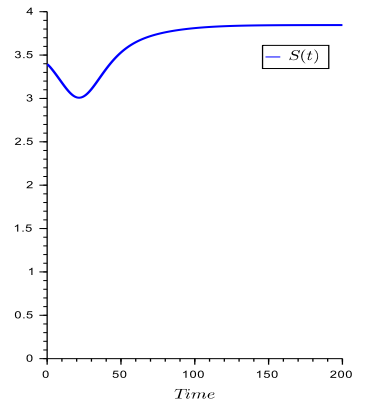

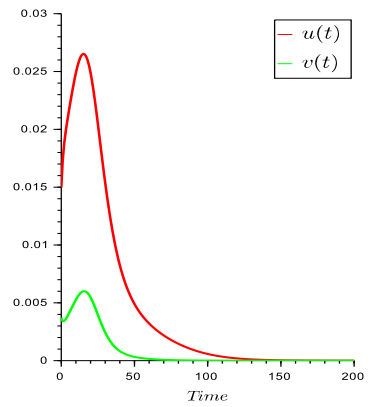

(b)

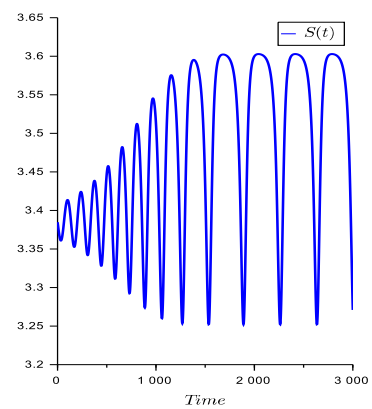

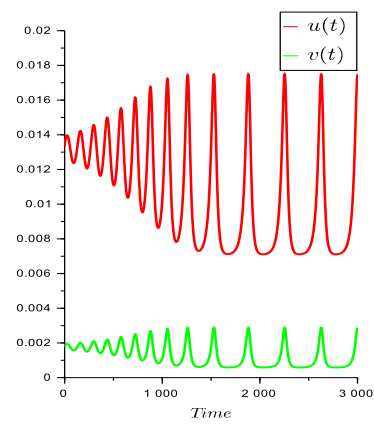

FIG. 7. Case $\left.S_{i n}=3.846 \in\right] S_{i n}^{2}, S_{i n}^{h_{1}}\left[:\right.$ bi-stability with convergence either to $E_{0}(a)$ or limit cycle when the oscillations are sustained (b).

For $\left.S_{i n} \in\right] S_{i n}^{2}, S_{i n}^{h_{1}}\left[\right.$, the bi-stability is transferred between $E_{0}$ and the limit cycle instead of $E_{0}$ and $E_{1}$. To detect the limit cycle, we take an initial condition close enough to positive steady state $E_{1}$ such that the convergence radius is small enough. Figure 7(a) shows the convergence to the washout steady state $E_{0}$ for an initial condition in a neighborhood of the saddle focus $E_{1}$ of size order $\varepsilon=2 \times 10^{-3}$ while Figure 7(b) clearly shows the trajectory starting from a neighborhood of $E_{1}$ of size order $\varepsilon=10^{-4}$ is approaching a limit cycle as time goes where the system exhibits sustained oscillations, which implies that the limit cycle is stable. All these facts tell us that a stable limit cycle is bifurcated from the steady state $E_{1}$ as the control parameter $S_{i n}$ passes through its critical value $S_{i n}^{2}$. 
(a)

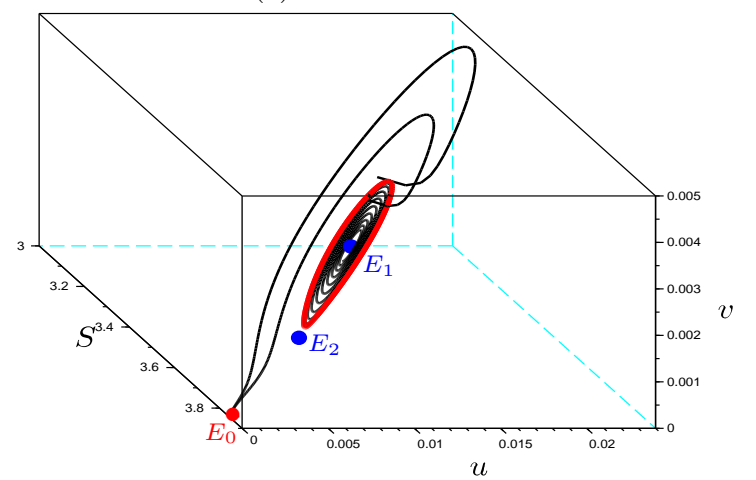

(b)

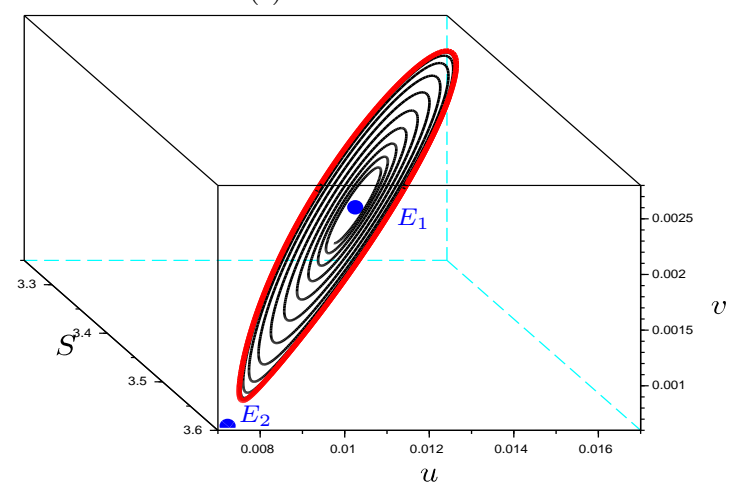

FIG. 8. Case $\left.S_{i n}=3.846 \in\right] S_{i n}^{2}, S_{i n}^{h_{1}}\left[:(a)\right.$ bi-stability of $E_{0}$ and limit cycle; (b) a magnification of limit cycle in the three-dimensional space $(S, u, v)$.

In Figure 8, the three-dimensional phase space shows the bi-stability with convergence to $E_{0}$ for two initial conditions in a neighborhood of $E_{1}$ of size order $\varepsilon=10^{-3}$ and $\varepsilon=1.5 \times 10^{-3}$, respectively, or limit cycle for the same initial condition as those in Figure 7(b). A magnification in the three-dimensional space shows more clearly the convergence toward the limit cycle. When $\left.S_{i n} \in\right] S_{i n}^{h_{1}}, S_{i n}^{h_{2}}[$, the numerical simulations can show the global convergence toward the washout steady state $E_{0}$ from any positive initial condition (see Figure 9).
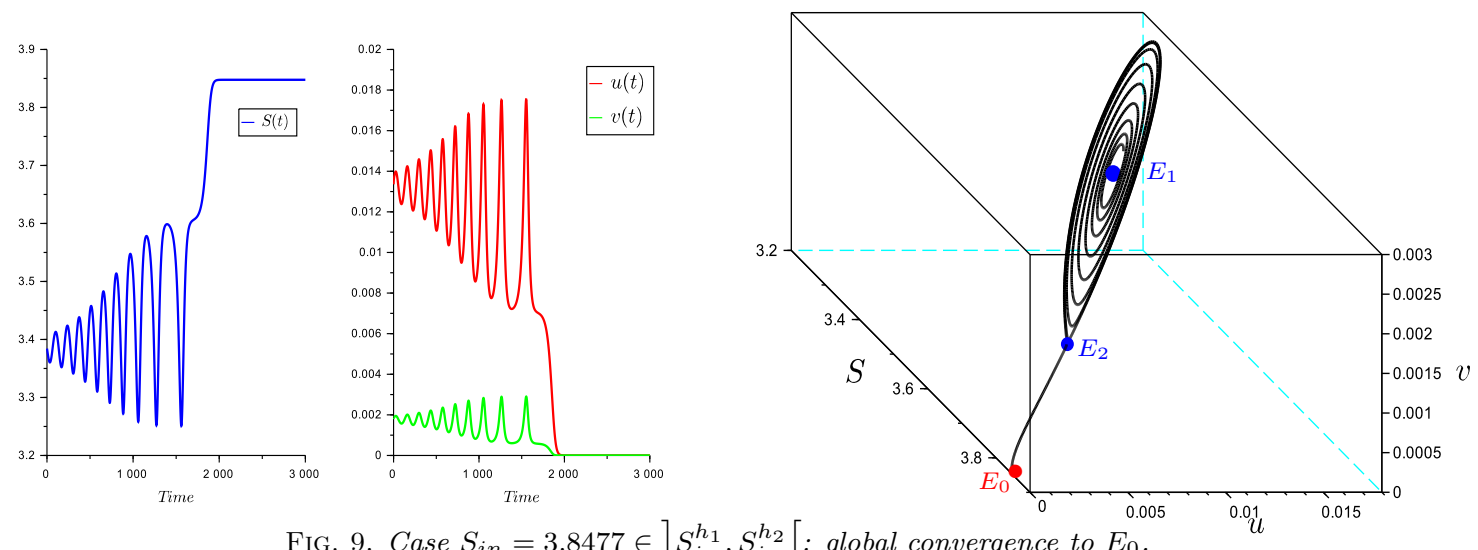

FIG. 9. Case $\left.S_{i n}=3.8477 \in\right] S_{i n}^{h_{1}}, S_{i n}^{h_{2}}\left[:\right.$ global convergence to $E_{0}$.

Figure 10 gives the time course and the phase portrait for $\left.S_{i n} \in\right] S_{i n}^{h_{2}}, \lambda_{u}[$ and shows that the system exhibits bi-stability with convergence either to washout steady state $E_{0}$ for an initial condition in a neighborhood of $E_{1}$ of size order $\varepsilon=2.1 \times 10^{-3}$ (a) or to the stable limit cycle for an initial condition in a neighborhood of $E_{1}$ of size order $\varepsilon=10^{-4}$ (b). In addition, the period asymptotes to infinity at a finite value of the bifurcation parameter $S_{i n}^{h_{2}}$. The three-dimensional phase plot shows the bi-stability where the blue trajectory tends to $E_{0}$ and the black trajectory tends to the red limit cycle (see Figure 10(c)).

The numerical simulations can show the global convergence toward the limit cycle from any positive initial condition and the oscillatory coexistence with constant amplitude and frequency over the time (see Figure 11).

Hence the sensitivity of flocculation model behavior in mortality of species and their effect on the occurrence of limit cycles via supercritical bifurcation. All these features cannot occur in cases 1 and 2 of (3.5), that is, $D_{u} \leq D$ and $D_{v} \leq D$, where there may be coexistence only around a positive steady state and not a limit cycle. 
(a)

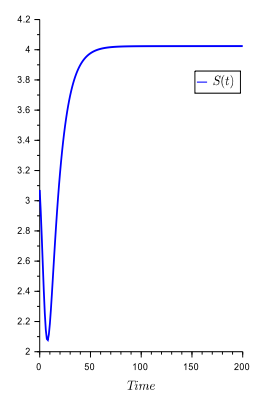

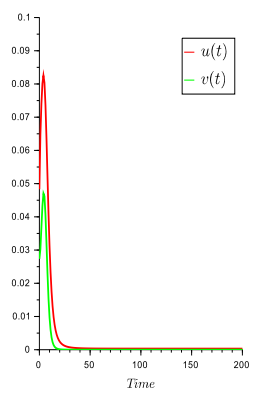

(b)

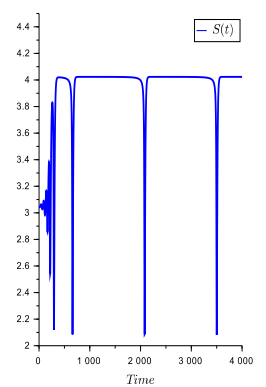

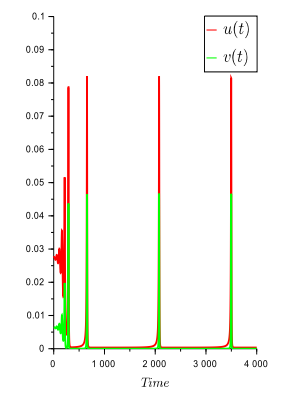

(c)

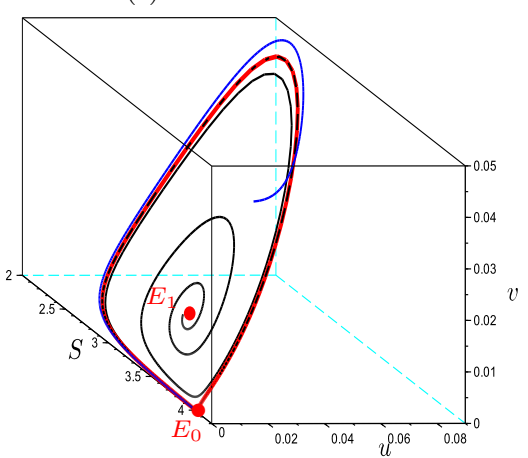

FIG. 10. Case $\left.S_{i n}=4.03468 \in\right] S_{i n}^{h_{2}}, \lambda_{u}\left[:\right.$ bi-stability and occurrence of limit cycle with convergence either to $E_{0}$ (a) or limit cycle (b). The limit cycle in the three-dimensional space $(S, u, v)$.
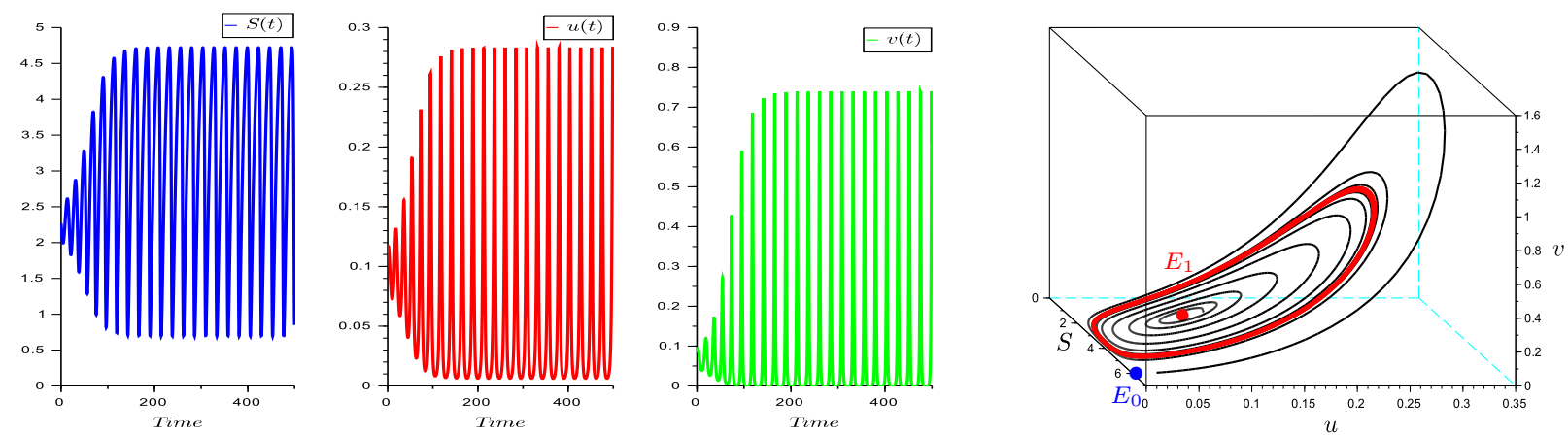

FIG. 11. Case $\left.S_{i n}=6.2 \in\right] \lambda_{u}, S_{i n}^{3}[:$ global convergence to limit cycle.

4.2. Occurrence of limit cycle: case 3 when $D_{v}<D<D_{u}$. In the previous section, we have shown the occurrence of limit cycles in the case where $D<D_{v}<D_{u}$. In what follows, we show that the two conditions $D<D_{u}$ and $D<D_{v}$ are not necessary and only one of these conditions is sufficient to destabilize the system. More precisely, we show the appearance of limit cycles in the third case of (3.5) when $D_{v}<D<D_{u}$ by finding a set of parameters such that $c_{4}$ can change its sign. Parameter values are given in Table 1 where we modified only the value of $D_{v}$ compared to the previous case $D<D_{v}<D_{u}$. In this case $\left(D_{v}<D<D_{u}\right)$, we have $H^{\prime}\left(S^{*}\right)<-D$ for all $\left.S^{*} \in I=\right] \lambda_{v}, \min \left(\lambda_{u}, \lambda_{b}\right)[$. Figure 12(a) illustrates the change of stability of $E_{1}$ according to values of $S$ at steady state when $c_{4}(S)$ changes sign at $S^{i}, i=1,2$, which is solution of the equation $c_{4}(S)=0$. The numerical simulations show that the Jacobian matrix of system (1.4) at $E_{1}$ has one negative eigenvalue and one pair of complex-conjugate eigenvalues that crosses the imaginary axis at $S_{i n}^{1}$ from negative half plane to positive half plane by increasing the control parameter $S_{i n}$ from $\lambda_{b}$. Then, it returns to the negative half plane by crossing the imaginary axis at $S_{i n}^{2}$ (see Figure 12(b)). Depending on the control parameter $S_{i n}$, one has the following change of stability:

- For $S_{i n} \in\left[0, \lambda_{b}\left[\right.\right.$, there exists a unique steady state which is the washout $E_{0}$ and it is LES.

- For $S_{i n}=\lambda_{b}, E_{1}$ appears stable node through a transcritical bifurcation while $E_{0}$ becomes a saddle point (see Figure 12(c)).

- For $\left.S_{i n} \in\right] \lambda_{b}, S_{i n}^{1}\left[, E_{1}\right.$ is LES where $c_{4}\left(S^{*}\right)>0$ when $\left.S^{*} \in\right] S^{1}, \lambda_{b}[($ see Figure 12(a)).

- For $\left.S_{i n} \in\right] S_{i n}^{1}, S_{i n}^{2}\left[, E_{1}\right.$ becomes unstable where $c_{4}<0$ when $\left.S^{*} \in\right] S^{2}, S^{1}[$.

- For $\left.S_{i n} \in\right] S_{i n}^{2},+\infty\left[, E_{1}\right.$ changes its stability and becomes LES where $c_{4}\left(S^{*}\right)>0$ when $S^{*} \in$ ]$\lambda_{v}, S^{2}[$.

The projection of the $\omega$-limit set in variable $S$ according to $S_{i n}$ reveals the appearance and disappearance of limit cycles through two supercritical Hopf bifurcations (see Figure 12(c)).

The numerical simulations shown in Figure 12 have been obtained with $D_{u}>D$ and $D_{v}<D$. 

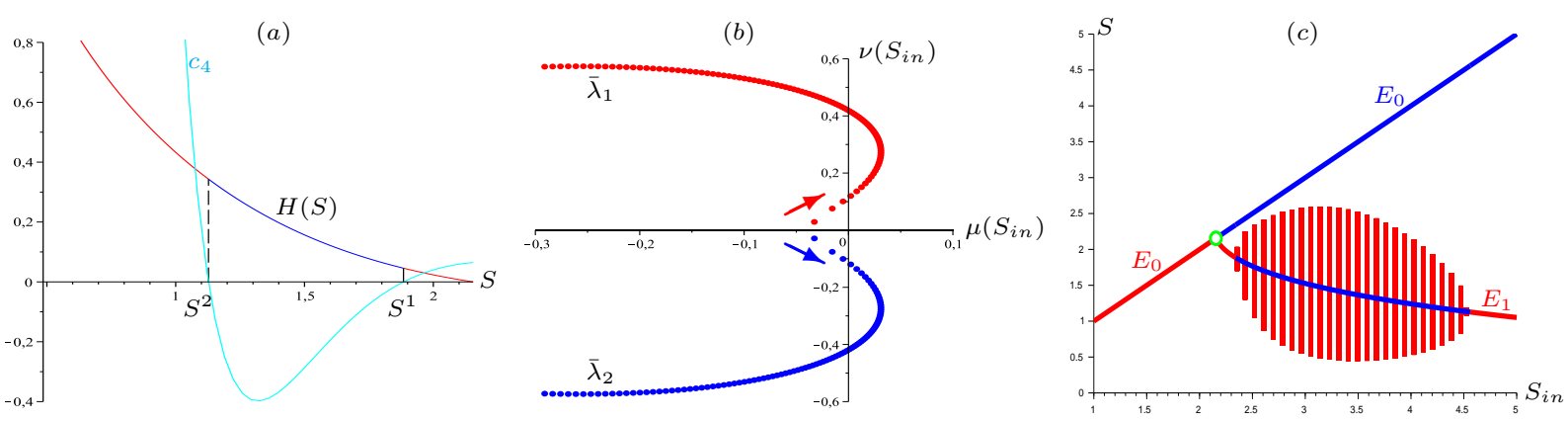

FIG. 12. (a) Change of sign of $c_{4}$ and the corresponding stability of $E_{1}$ on the red or blue curve of function $H(\cdot)$ where $\lambda_{b} \approx 2.155, S^{1} \approx 1.884$ (or $S_{i n}^{1} \approx 2.342$ ) and $S^{2} \approx 1.127$ (or $S_{i n}^{2} \approx 4.561$ ). (b) Variation of a pair of complex-conjugate eigenvalues as $S_{i n}$ increases. (c) Projection of the $\omega$-limit set in variable $S$ as a function of $S_{i n}$. A green open circle represents a transcritical bifurcation.

Therefore, the mortality of isolated bacteria, which is necessary to have $D_{u}>D$, can lead to their coexistence with bacteria in flocs around a stable limit cycle. Thus, the condition $\min \left(D_{u}, D_{v}\right) \leq D$ does not imply $c_{4}>0$ since, for instance the condition $D_{v}<D<D_{u}$ permits to destabilize the system.

4.3. Multiplicity of positive steady states. In this section, we illustrate the bi-stability and the multiplicity of positive steady states of flocculation model (1.4) in case 2 of (3.5). When the growth rates are of Monod-type (4.1), the equation $D\left(S_{i n}-S\right)=H(S)$ is equivalent to a polynomial equation of fifth degree. Therefore, there is at most five solutions of this equation. The positive steady states correspond to solutions which are in the interval $I$. We succeeded in finding a set of parameters with 3 solutions at most in this interval. The numerical simulations illustrate the results of Proposition 2.6 and Proposition 2.7, which are obtained for the Monod-type growth rates (4.1). All parameter values used in this section are summarized in Table 1. Figure 13 illustrates the case $\lambda_{u}<S_{i n}<\lambda_{v}$ where there exists a unique positive steady state

$$
E_{1} \simeq(3.37,0.98,1.38)
$$

which is LES. The numerical simulation shows the global convergence to the positive steady state $E_{1}$ for any positive initial condition. Figure 14 illustrates the case $\lambda_{u}<\lambda_{v}<S_{i n}$ with three positive steady

(a)

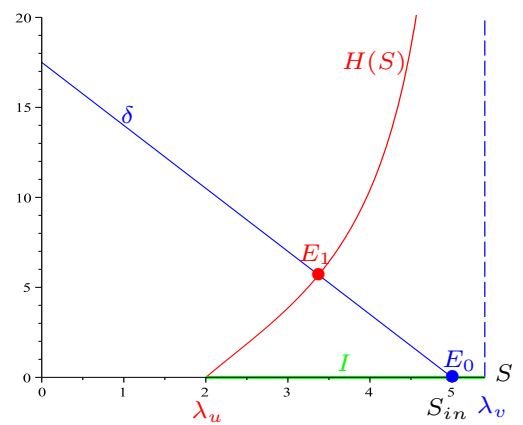

(b)

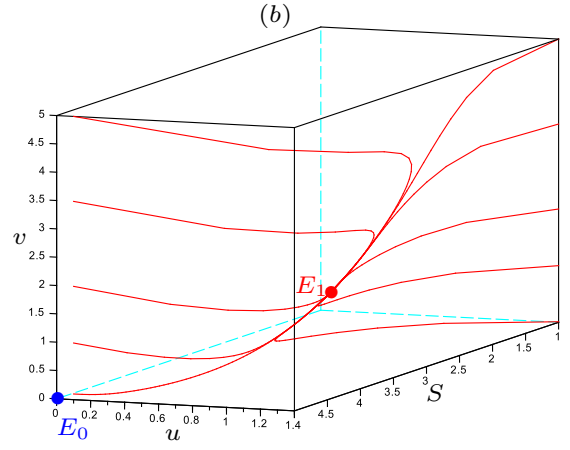

FIG. 13. The case $\lambda_{u}=2<S_{i n}=5<\lambda_{v}=5.4$ : a unique positive steady state $E_{1}$ and global convergence to $E_{1}$.

states

$$
E_{1} \simeq(3.06,12.11,157.46), \quad E_{2} \simeq(5.17,8.53,524.30) \text { and } E_{3} \simeq(8.81,2.64,1086.32) .
$$

The numerical simulations show the bi-stability with two basins of attraction, one toward $E_{1}$ and the other toward $E_{3}$ which are stable nodes. These two basins are separated by the stable manifold of saddle point $E_{2}$. As it was proved in Proposition 2.7, the steady state $E_{1}$ promotes planktonic biomass $u$ and 
(a)

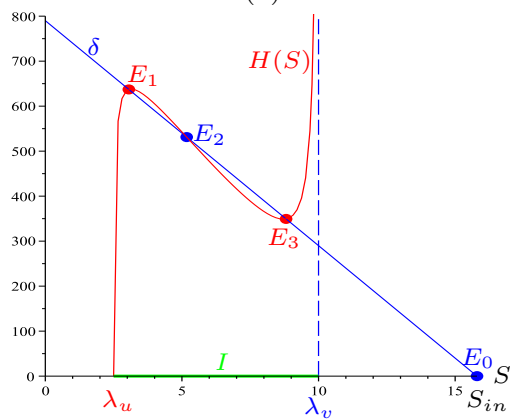

(b)

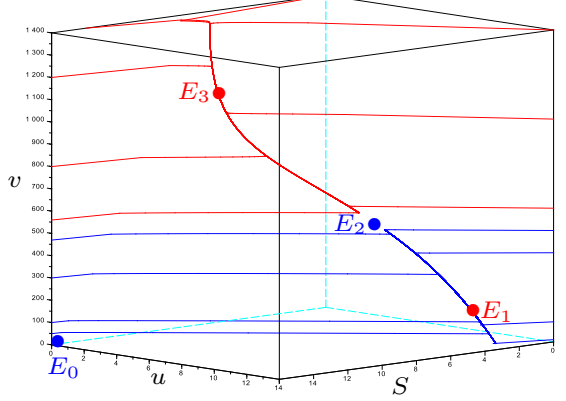

FIG. 14. The case $\lambda_{u}=2.5<\lambda_{v}=10<S_{\text {in }}=15.8$ : three positive steady states and bi-stability.

$E_{3}$ promotes attached biomass $v$. Figure 15 illustrates the case $\lambda_{v}<S_{i n}<\lambda_{b}<\lambda_{u}$ with two positive steady states

$$
E_{1} \simeq(1.37,0.19,0.62) \quad \text { and } \quad E_{2} \simeq(1.73,0.07,0.1) .
$$

The numerical simulations show the bi-stability with two basins of attraction, which are separated by the stable manifold of saddle point $E_{2}$. One basin of attraction attracts the solutions to the positive steady state $E_{1}$ and another to the washout steady state $E_{0}$. Figure 16 illustrates the case $S_{i n}>\lambda_{b}$ with a

(a)

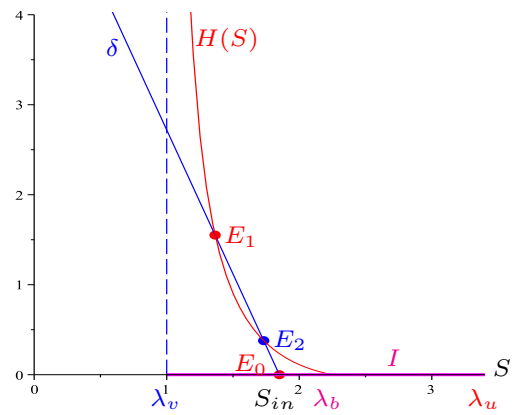

(b)

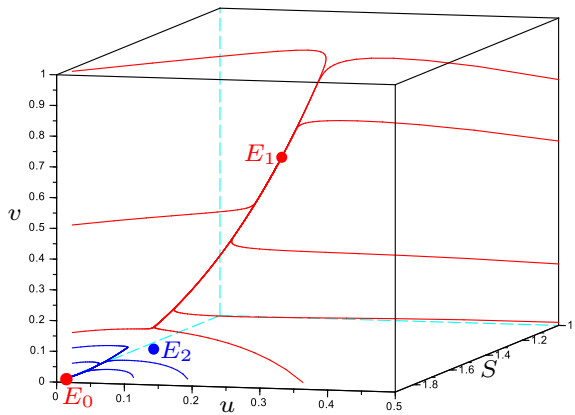

FIG. 15. The case $\lambda_{v}=1<S_{i n}=1.85<\lambda_{b}=2.25<\lambda_{u}=3.4$ : two positive steady states and bi-stability.

unique positive steady state

$$
E_{1} \simeq(1.15,0.3,2.54)
$$

The numerical simulations show the global convergence to the positive steady state $E_{1}$ for any positive initial condition. Figure 17 illustrates the case $S_{i n}>\lambda_{u}>\lambda_{v}$ with three positive steady states

(a)

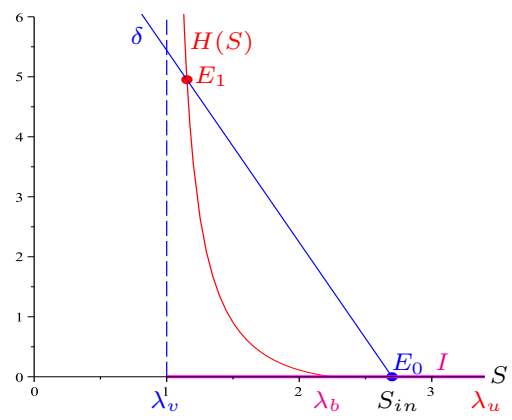

(b)

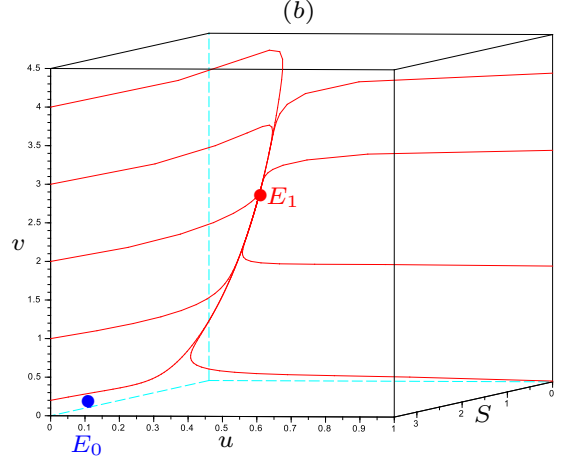

FIG. 16. The case $\lambda_{v}=1<\lambda_{b}<S_{i n}=2.7<\lambda_{u}=3.4$ : a unique positive steady state $E_{1}$ and global convergence to $E_{1}$. 


$$
E_{1} \simeq(3.31,2.23,27.08), \quad E_{2} \simeq(3.98,1.67,4.12) \quad \text { and } \quad E_{3} \simeq(4.39,0.63,0.24) .
$$

The numerical simulations show the bi-stability with two basins of attraction, one to the positive steady state $E_{1}$ and the other to the positive steady state $E_{3}$ which are stable nodes. These two basins are separated by the stable manifold of saddle point $E_{2}$. As it was proved in Proposition 2.7, the steady state $E_{1}$ promotes simultaneously two biomass $u$ and $v$. Figure 18 illustrates the case $\lambda_{v}<S_{i n}<\lambda_{u}$ with no

$(a)$

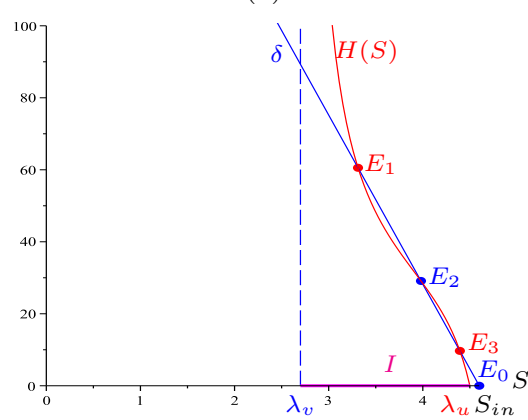

(b)

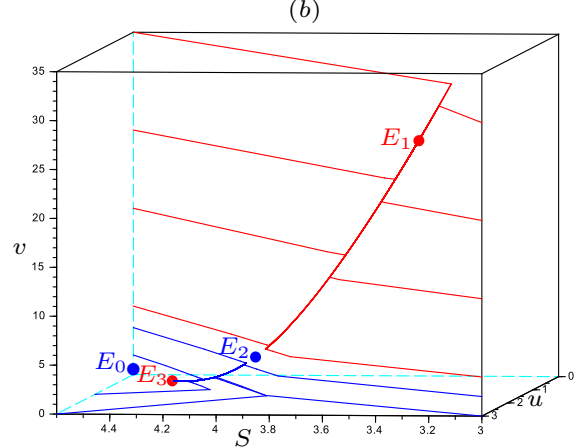

FIG. 17. The case $\lambda_{v}=2.7<\lambda_{u}=4.5<S_{i n}=4.6$ : existence of three positive steady states and bi-stability.

positive steady state. The numerical simulations show the global convergence toward the washout steady state $E_{0}$.

(a)

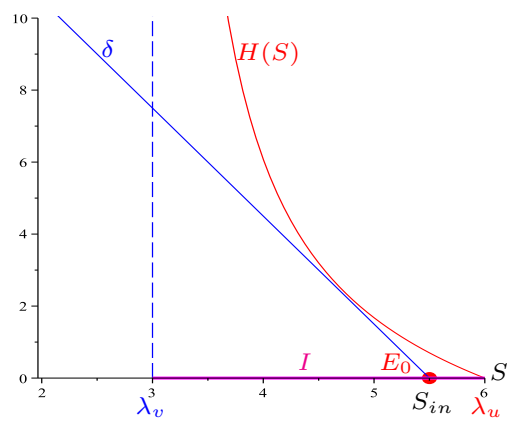

(b)

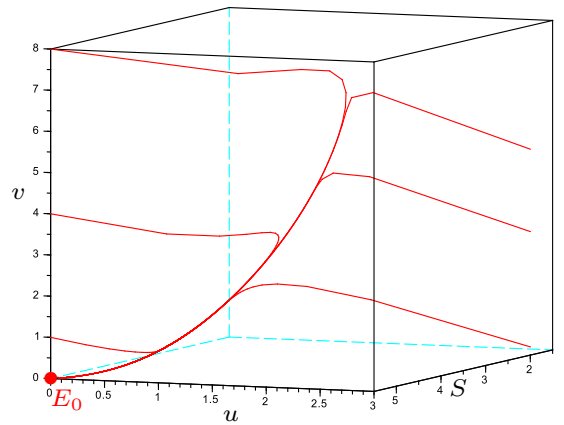

FIG. 18. The case $\lambda_{v}=3<S_{i n}=5.5<\lambda_{u}=6$ : global convergence toward $E_{0}$.

5. Conclusion. In this work, we have analyzed mathematically and through numerical simulations a model of the chemostat with three nonlinear differential equations where one species is present in two forms, isolated and attached with the presence of a single growth-limiting resource. The new feature was that maintenance terms are added to depletion or removal rates in order to give a complete analysis of flocculation model (1.4).

To our knowledge, our study is the first attempt to bring out the common effects of the flocculation phenomenon and mortality in the coexistence around a stable limit cycle. Depending on the two control parameters $S_{\text {in }}$ and $D$, the flocculation model may exhibit sustained oscillations and the occurrence of stable limit cycles via supercritical Hopf bifurcations.

More precisely, when $D_{v}<D_{u}$, we show that the system may exhibit bi-stability with multiplicity of coexistence steady states that can bifurcate through saddle-node bifurcations or transcritical bifurcations. Whereas, the bi-stability could occur in the classical chemostat model (1.1) only when the growth rate is non-monotonic. If, in addition $D_{u} \leq D$, the coexistence of planktonic and attached bacteria may be only around a positive steady state.

Considering the mortality of isolated and aggregated bacteria $\left(D<D_{v}<D_{u}\right)$, we have identified that the phase portraits may be very rich. More precisely, the one-parameter bifurcation diagram for 
model (1.4) shows the effect of control parameter $S_{i n}$ on the behavior of the system. For small enough $S_{i n}$, there is exclusion of planktonic and attached species. Increasing $S_{i n}$, system (1.4) undergoes Hopf bifurcations at the positive steady state $E_{1}$. Furthermore, this system may exhibit bi-stability with convergence either to a stable limit cycle or to the washout steady state. The disappearance of stable limit cycles can be either by supercritical Hopf bifurcations or homoclinic bifurcations. For large enough $S_{i n}$, there is global convergence to the positive steady state or to a stable limit cycle. However, we have shown that the mortality of planktonic or attached species (for instance $D_{v}<D<D_{u}$ ) suffices to ensure the coexistence around a stable limit cycle.

Our findings on the destabilization by of the positive steady state are similar to those in $[3,19]$. In [19], the steady state of a trophic chain (prey-predator) in a chemostat can be destabilized by mortality where stable limit cycles and multiple chaotic attractors are found. The maintenance (or decay) rate coefficients considered in a tri-trophic food chain model can cause destabilization of system when the operating diagram shows local and global bifurcations of steady states and of limit cycles [3].

Our results show that the mortality of the species is necessary for the emergence of stable limit cycles in the flocculation model (1.4). This is mainly due to the joined effect of mortality and flocculation. However, in the flocculation model introduced in [11], without mortality $\left(D_{u}=D_{v}=D\right)$, it has been shown that the model can have unstable limit cycles with a non-monotonic growth rate of planktonic bacteria, see [7]. It is the joined effect of flocculation and inhibition by the substrate on the growth that was the cause of the appearance of unstable limit cycles. Adding a second species to the model, where only the most efficient species makes flocs, the model does produce oscillations with emergence of a stable limit cycle. Therefore, the properties of the model depend highly on the flocculation phenomenon through the attachment and detachment velocities $A(\cdot)$ and $B(\cdot)$ respectively in (1.3) and should be carefully discussed with the biologists.

As it was noticed in the introduction, a more general choice for the specific velocity $A(\cdot)$ of aggregation, which is still coherent with Smoluchowski's modeling of flocculation [10,35], should be $A(\cdot)=a_{u} u+a_{v} v$ with distinct coefficients $a_{u}$ and $a_{v}$. The particular case $a_{v}=0$ corresponds to the model in [11] and the particular case $a_{u}=a_{v}$ corresponds to the model in the present paper. The study of the robustness of the model with respect to this variation, or with other terms tainted with some light non-linearity, is an important question that deserves further attention and will be the object of a future work.

Thereby, the flocculation models are sensitive to mortality of species which is neglected in the literature. The behavior of the system is richer with coexistence, bi-stability, multiplicity of positive steady states, and emergence of stable limit cycles. This last feature cannot occur in the flocculation model without mortality what confirms the output sensitivity and the importance of mortality in biological systems. All these bifurcations enrich notably the dynamic behavior of the analyzed flocculation model (1.4).

\section{Appendix A. Proofs.}

A.1. First proofs. In this Appendix, we give the proofs of the results given in section 2. Proof of Proposition 2.3. Since

$$
S=0 \Rightarrow \dot{S}=D S_{\text {in }}>0,
$$

then no trajectory can leave the positive octant $\mathbb{R}_{+}^{3}$ by crossing the boundary face $S=0$. In addition, whenever $u=0$ with $v>0$, then $\dot{u}=b v>0$. Similarly, whenever $v=0$ with $u>0$, then $\dot{v}=a u^{2}>0$. Hence, the vector field points inside

$$
\Theta=\left\{(S, u, v) \in \mathbb{R}^{3}: S>0, u>0, v>0\right\}
$$

along the whole boundary of $\Theta$ without the horizontal semi-axis

$$
\Gamma=\{S \geq 0, u=v=0\},
$$

which is invariant under the system (1.4) because the function

$$
t \rightarrow(S(t), u(t), v(t))=\left(S_{\text {in }}+\left(S(0)-S_{\text {in }}\right) e^{-D t}, 0,0\right)
$$


is a solution of (1.4). By uniqueness of solutions, $\Gamma$ cannot be reached in finite time by trajectories for which $u>0$ or $v>0$. Therefore, the solutions remain non-negative.

Let $z=S+u+v$. The sum of the three equations of (1.4) yields

$$
\dot{z}(t)=D S_{i n}-D S(t)-D_{u} u(t)-D_{v} v(t) \leq D_{\min }\left(\frac{D}{D_{\min }} S_{i n}-z(t)\right) .
$$

Using Gronwall's Lemma, we obtain

$$
z(t) \leq \frac{D}{D_{\min }} S_{\text {in }}+\left(z(0)-\frac{D}{D_{\min }} S_{\text {in }}\right) e^{-D_{\min } t} \quad \text { for all } \quad t \geq 0 .
$$

We deduce that

$$
z(t) \leq \max \left(z(0), \frac{D}{D_{\min }} S_{\text {in }}\right) \quad \text { for all } t \geq 0 .
$$

Therefore, the solutions of (1.4) are positively bounded and are defined for all $t \geq 0$. From (A.1), it can be deduced that the set $\Omega$ is positively invariant and is a global attractor for (1.4).

Proof of Proposition 2.7. A straightforward calculation shows that

$$
H(S)=\frac{\varphi(S)(\psi(S)-b)\left[D_{u} \psi(S)-D_{v} \varphi(S)\right]}{a[\psi(S)-\varphi(S)] \psi(S)} .
$$

From (2.6) and (A.2), it follows that

$$
\frac{\varphi\left(S^{*}\right)\left(\psi\left(S^{*}\right)-b\right)}{a\left[\psi\left(S^{*}\right)-\varphi\left(S^{*}\right)\right]}=\frac{D\left(S_{i n}-S^{*}\right) \psi\left(S^{*}\right)}{D_{u} \psi\left(S^{*}\right)-D_{v} \varphi\left(S^{*}\right)} .
$$

Therefore, the expressions (2.9) and (2.10) can be rewritten also as follows:

$$
u^{*}=U_{1}\left(S^{*}\right) \quad \text { with } \quad U_{1}(S):=\frac{D\left(S_{i n}-S\right) \psi(S)}{D_{u} \psi(S)-D_{v} \varphi(S)}
$$

and

$$
v^{*}=V_{1}\left(S^{*}\right) \quad \text { with } \quad V_{1}(S)=\frac{D\left(S_{i n}-S\right) \varphi(S)}{D_{v} \varphi(S)-D_{u} \psi(S)}
$$

We show that

1. If $\lambda_{u}<\lambda_{v}$, then $U_{1}(\cdot)$ is strictly decreasing on $I \cap\left[0, S_{i n}\right]$ and $V(\cdot)$ is strictly increasing on $I$.

2. If $\lambda_{v}<\lambda_{u}$, then $U$ and $V$ are strictly decreasing on $I$ and $V_{1}$ is strictly decreasing $I \cap\left[0, S_{i n}\right]$. From (2.9) and (A.3), a simple calculation yields that

$U_{1}^{\prime}(S)=D \frac{-\psi\left(D_{u} \psi-D_{v} \varphi\right)-g^{\prime} D_{v} \varphi\left(S_{i n}-S\right)+f^{\prime} D_{v} \psi\left(S_{i n}-S\right)}{\left(D_{u} \psi-D_{v} \varphi\right)^{2}}, \quad U^{\prime}(S)=\frac{f^{\prime} \psi(\psi-b)+g^{\prime} \varphi(b-\varphi)}{a(\psi-\varphi)^{2}}$.

Therefore, if $\lambda_{u}<\lambda_{v}$, then $U_{1}^{\prime}(S)$ is negative on $I \cap\left[0, S_{i n}\right]$ and if $\lambda_{v}<\lambda_{u}$, then $U^{\prime}(S)$ is negative on $I$. From (2.10) and (A.4), a direct calculation shows that

$$
\begin{gathered}
V_{1}^{\prime}(S)=D \frac{-\varphi\left(D_{v} \varphi-D_{u} \psi\right)-f^{\prime} D_{u} \psi\left(S_{i n}-S\right)+g^{\prime} D_{u} \varphi\left(S_{i n}-S\right)}{\left(D_{v} \varphi-D_{u} \psi\right)^{2}}, \\
V^{\prime}(S)=\frac{-f^{\prime}[\varphi \psi(\psi-b)](2 \psi-\varphi)+g^{\prime} \varphi^{2}(\psi-b)(2 \psi-\varphi)}{a(\psi-\varphi)^{2} \psi^{2}} .
\end{gathered}
$$


If $\lambda_{u}<\lambda_{v}$, then $V^{\prime}(S)$ is positive on $I$ and if $\lambda_{v}<\lambda_{u}$, then $V_{1}^{\prime}(S)$ is negative on $I \cap\left[0, S_{\text {in }}\right]$ and $V^{\prime}(S)$ is negative on $I$. Let $S^{*}<S^{* *}$. Therefore, if $\lambda_{u}<\lambda_{v}$, then

$$
u^{*}=U_{1}\left(S^{*}\right)>u^{* *}=U_{1}\left(S^{* *}\right) \quad \text { and } \quad v^{*}=V\left(S^{*}\right)<v^{* *}=V\left(S^{* *}\right) .
$$

Furthermore, if $\lambda_{v}<\lambda_{u}$ then

$$
u^{*}=U\left(S^{*}\right)>u^{* *}=U\left(S^{* *}\right) \text { and } v^{*}=V_{1}\left(S^{*}\right)>v^{* *}=V_{1}\left(S^{* *}\right) .
$$

This completes the proof.

A.2. The sign of $c_{1}$. In this section, we consider the sign of the coefficient $c_{1}$.

Proof of Lemma 3.2. From the second equation of (2.1), we have

$\varphi\left(S^{*}\right) u^{*}-a\left(u^{*}+v^{*}\right) u^{*}+b v^{*}=\varphi\left(S^{*}\right) u^{*}-a\left(2 u^{*}+v^{*}\right) u^{*}+a\left(u^{*}\right)^{2}+b v^{*}=-m_{22} u^{*}+a\left(u^{*}\right)^{2}+b v^{*}=0$.

Hence

$$
m_{22}=a u^{*}+b \frac{v^{*}}{u^{*}}>0
$$

From the third equation of (2.1), we have

$$
\psi\left(S^{*}\right) v^{*}+a\left(u^{*}+v^{*}\right) u^{*}-b v^{*}=-m_{33} v^{*}+a\left(u^{*}\right)^{2}=0 .
$$

and therefore,

$$
m_{33}=a \frac{\left(u^{*}\right)^{2}}{v^{*}}>0
$$

Thus, all $m_{i j}$ are positive for all $i, j=1, \ldots, 3$ with $(i, j) \neq(2,3)$. Since $m_{i i}>0, i=1, \ldots, 3$, then $c_{1}=\sum_{i=1}^{3} m_{i i}>0$.

A.3. The sign of $c_{3}$. We study the sign of $c_{3}$ is a more general context. Consider the following system of differential equations

$$
\left\{\begin{array}{l}
\dot{x}_{0}=f_{0}\left(x_{0}, x_{1}, x_{2}\right) \\
\dot{x}_{1}=f_{1}\left(x_{0}, x_{1}, x_{2}\right) \\
\dot{x}_{2}=f_{2}\left(x_{0}, x_{1}, x_{2}\right) .
\end{array}\right.
$$

Let $x^{*}=\left(x_{0}^{*}, x_{1}^{*}, x_{2}^{*}\right)$ be a steady state, and let

$$
J=\left[\begin{array}{lll}
a_{00} & a_{01} & a_{02} \\
a_{10} & a_{11} & a_{12} \\
a_{20} & a_{21} & a_{22}
\end{array}\right]
$$

where

$$
a_{i j}=\frac{\partial f_{i}}{\partial x_{j}}\left(x^{*}\right), \quad i=0,1,2, \quad j=0,1,2
$$

be the Jacobian matrix of (A.5) at $x^{*}$. The steady state $x^{*}=\left(x_{0}^{*}, x_{1}^{*}, x_{2}^{*}\right)$ is a solution of the set of equations

$$
\left\{\begin{array}{l}
0=f_{0}\left(x_{0}, x_{1}, x_{2}\right) \\
0=f_{1}\left(x_{0}, x_{1}, x_{2}\right) \\
0=f_{2}\left(x_{0}, x_{1}, x_{2}\right) .
\end{array}\right.
$$

We solve this set of equations in the following manner: 
1. We first solve the second and third equations $f_{1}\left(x_{0}, x_{1}, x_{2}\right)=0$ and $f_{2}\left(x_{0}, x_{1}, x_{2}\right)=0$ which are assumed to define $x_{1}$ and $x_{2}$ as functions of $x_{0}$, that is to say, there exists $x_{0} \mapsto\left(X_{1}\left(x_{0}\right), X_{2}\left(x_{0}\right)\right)$ such that

$$
f_{1}\left(x_{0}, X_{1}\left(x_{0}\right), X_{2}\left(x_{0}\right)\right)=0 \text { and } f_{2}\left(x_{0}, X_{1}\left(x_{0}\right), X_{2}\left(x_{0}\right)\right)=0 .
$$

2. Then, we replace $x_{1}$ by $X_{1}\left(x_{0}\right)$ and $x_{2}$ by $X_{2}\left(x_{0}\right)$ in the first equation to obtain

$$
h\left(x_{0}\right)=0, \quad \text { where } h\left(x_{0}\right)=f_{0}\left(x_{0}, X_{1}\left(x_{0}\right), X_{2}\left(x_{0}\right)\right)
$$

which is assumed to have a solution $x_{0}^{*}$.

3. Therefore, $x_{1}^{*}=X_{1}\left(x_{0}^{*}\right)$ and $x_{2}^{*}=X_{2}\left(x_{0}^{*}\right)$ define the steady state $x^{*}=\left(x_{0}^{*}, x_{1}^{*}, x_{2}^{*}\right)$.

Lemma A.1. Assume that $\Delta:=a_{11} a_{22}-a_{12} a_{21} \neq 0$, then we have the following formula

$$
\operatorname{det}(J)=h^{\prime}\left(x_{0}^{*}\right) \Delta .
$$

Proof. Deriving (A.6) with respect to $x_{0}$ gives the following formulas

$$
\left\{\begin{array}{l}
\frac{\partial f_{1}}{\partial x_{0}}+\frac{\partial f_{1}}{\partial x_{1}} X_{1}^{\prime}\left(x_{0}\right)+\frac{\partial f_{1}}{\partial x_{2}} X_{2}^{\prime}\left(x_{0}\right)=0 \\
\frac{\partial f_{2}}{\partial x_{0}}+\frac{\partial f_{2}}{\partial x_{1}} X_{1}^{\prime}\left(x_{0}\right)+\frac{\partial f_{2}}{\partial x_{2}} X_{2}^{\prime}\left(x_{0}\right)=0
\end{array}\right.
$$

where the partial derivatives of $f_{1}$ and $f_{2}$ are evaluated in $\left(x_{0}, X_{1}\left(x_{0}\right), X_{2}\left(x_{0}\right)\right)$. For $x_{0}=x_{0}^{*}$, system (A.8) becomes

$$
\left\{\begin{array}{l}
a_{10}+a_{11} X_{1}^{\prime}\left(x_{0}^{*}\right)+a_{12} X_{2}^{\prime}\left(x_{0}^{*}\right)=0 \\
a_{20}+a_{21} X_{1}^{\prime}\left(x_{0}^{*}\right)+a_{22} X_{2}^{\prime}\left(x_{0}^{*}\right)=0
\end{array}\right.
$$

Using $\Delta \neq 0$, we have

$$
X_{1}^{\prime}\left(x_{0}^{*}\right)=-\frac{a_{10} a_{22}-a_{20} a_{12}}{\Delta}, \quad X_{2}^{\prime}\left(x_{0}^{*}\right)=-\frac{a_{20} a_{11}-a_{10} a_{21}}{\Delta} .
$$

The development of the determinant of $J$ with respect to the first line gives

$$
\operatorname{det}(J)=a_{00} \Delta-a_{01}\left(a_{10} a_{22}-a_{20} a_{12}\right)+a_{02}\left(a_{10} a_{21}-a_{20} a_{11}\right) .
$$

Thus,

$$
\operatorname{det}(J)=\Delta\left(a_{00}+a_{01} X_{1}^{\prime}\left(x_{0}^{*}\right)+a_{02} X_{2}^{\prime}\left(x_{0}^{*}\right)\right) .
$$

By using expression (A.7) of $h\left(x_{0}\right)$, it follows that

$$
h^{\prime}\left(x_{0}\right)=\frac{\partial f_{0}}{\partial x_{0}}+\frac{\partial f_{0}}{\partial x_{1}} X_{1}^{\prime}\left(x_{0}\right)+\frac{\partial f_{0}}{\partial x_{2}} X_{2}^{\prime}\left(x_{0}\right) .
$$

Hence, $h^{\prime}\left(x_{0}^{*}\right)=a_{00}+a_{01} X_{1}^{\prime}\left(x_{0}^{*}\right)+a_{02} X_{2}^{\prime}\left(x_{0}^{*}\right)$. Therefore, $\operatorname{det}(J)=\Delta h^{\prime}\left(x_{0}^{*}\right)$.

Proof of Proposition 3.3. In the particular case of system (1.4), we have

$$
\left\{\begin{array}{l}
f_{0}\left(x_{0}, x_{1}, x_{2}\right)=D\left(S_{i n}-x_{0}\right)-f\left(x_{0}\right) x_{1}-g\left(x_{0}\right) x_{2} \\
f_{1}\left(x_{0}, x_{1}, x_{2}\right)=\left[f\left(x_{0}\right)-D_{u}\right] x_{1}-a\left(x_{1}+x_{2}\right) x_{1}+b x_{2} \\
f_{2}\left(x_{0}, x_{1}, x_{2}\right)=\left[g\left(x_{0}\right)-D_{v}\right] x_{2}+a\left(x_{1}+x_{2}\right) x_{1}-b x_{2} .
\end{array}\right.
$$

Since,

$$
h\left(x_{0}\right)=D\left(S_{i n}-x_{0}\right)-H\left(x_{0}\right), \quad \text { where } H\left(x_{0}\right)=f\left(x_{0}\right) X_{1}\left(x_{0}\right)+g\left(x_{0}\right) X_{2}\left(x_{0}\right) .
$$

it follows that

$$
h^{\prime}\left(x_{0}\right)=-D-H^{\prime}\left(x_{0}\right)
$$


Substituting $x_{1}^{*}$ and $x_{2}^{*}$ by their expressions $U\left(S^{*}\right)$ and $V\left(S^{*}\right)$ given by (2.9) and (2.10), respectively, in the expression of $\Delta$, a straightforward calculation shows that

$$
\Delta=-\varphi\left(x_{0}^{*}\right)\left(\psi\left(x_{0}^{*}\right)-b\right) .
$$

We conclude from Lemma A.1 that,

$$
c_{3}=-\operatorname{det}(J)=-\varphi\left(x_{0}^{*}\right)\left(\psi\left(x_{0}^{*}\right)-b\right)\left(D+H^{\prime}\left(x_{0}^{*}\right)\right) .
$$

A.4. The sign of $c_{4}$. In this section we consider the sign of the coefficient $c_{4}$.

Proof of Proposition 3.4. In the case 1, one has $D_{u} \leq D_{v}$. From Lemma Lemma 2.5, it follows that $\lambda_{u}<\lambda_{v}$. In the case 2, one has $D_{v}<D_{u}$. From Lemma Lemma 2.5, two cases must be distinguished: either $\lambda_{u}<\lambda_{v}$ or $\lambda_{u}>\lambda_{v}$. Straightforward calculations show that:

$$
c_{4}=D_{u} D f^{\prime} u^{*}+D g^{\prime} a u^{*} v^{*}+f^{\prime} \frac{\varphi(\psi-b)^{2}}{a(\psi-\varphi)^{2} \psi} F+g^{\prime} \frac{\varphi^{2}(\psi-b)}{a[(\psi-\varphi) \psi]^{2}} G+P_{1}
$$

where

$$
\begin{gathered}
F=2\left(D-D_{v}\right) \varphi \psi+\left(D_{v}-D\right) \varphi^{2}+\left(D_{u}-D\right) \psi^{2}+D \varphi \psi \\
G=\left(D-D_{v}\right) b \varphi(2 \psi-\varphi)+D \psi^{3}+\left(D_{u}-D\right) b \psi^{2}-D_{u} \varphi \psi^{2}+\left(D_{v}-D\right) \psi^{2} \varphi \\
P_{1}=D^{2}\left(m_{22} m_{33}\right)+D\left(f^{\prime} u^{*}+g^{\prime} v^{*}\right) m_{33}+\left(m_{22} m_{33}\right) c_{2} .
\end{gathered}
$$

From (3.3), $c_{2}$ can be written as follows:

$$
c_{2}=m_{12} m_{21}+m_{13} m_{31}+m_{11}\left(m_{22}+m_{33}\right)+m_{22} m_{33}-m_{32} a_{23} .
$$

Since $m_{22} m_{33}-m_{32} a_{23}=\Delta$, it follows from its expression (A.9) that:

$$
m_{22} m_{33}-m_{32} a_{23}=\Delta=-\varphi\left(S^{*}\right)\left[\psi\left(S^{*}\right)-b\right] .
$$

Let $\lambda_{u}<\lambda_{v}$. Thus, $\Delta$ is positive and so $c_{2}>0$. Consequently, $P_{1}$ is positive. In the case $1\left(D_{u} \leq D_{v} \leq D\right)$ and the case $2\left(D_{v}<D_{u} \leq D\right)$, it is easy to check $F<0$ and $G<0$ where $\psi<0<\varphi$ on $] \lambda_{u}, \lambda_{v}[$. Thus, one can conclude that $c_{4}>0$ in the first and the second cases when $\lambda_{u}<\lambda_{v}$.

Let $\lambda_{u}>\lambda_{v}$. Straightforward calculations show that:

$$
c_{4}=A+B+P_{2}
$$

where

$$
\begin{gathered}
A=D m_{12} m_{21}+m_{11}^{2} m_{22}-m_{21} m_{32} m_{13}, \quad B=D m_{13} m_{31}+m_{11}^{2} m_{33}-m_{31} a_{23} m_{12}, \\
P_{2}=\left(f^{\prime} u^{*}+g^{\prime} v^{*}\right) m_{12} m_{21}+\left(f^{\prime} u^{*}+g^{\prime} v^{*}\right) m_{13} m_{31}+m_{22}\left(c_{2}-m_{13} m_{31}\right)+m_{33}\left(c_{2}-m_{12} m_{21}\right) .
\end{gathered}
$$

From (A.11) and (A.12), one has

$$
c_{2}-m_{13} m_{31}=m_{12} m_{21}+m_{11}\left(m_{22}+m_{33}\right)+\varphi(b-\psi)>0,
$$

and

$$
c_{2}-m_{12} m_{21}=m_{13} m_{31}+m_{11}\left(m_{22}+m_{33}\right)+\varphi(b-\psi)>0 .
$$


Thus, $P_{2}>0$. On the one hand, we can write $m_{11}^{2}=D f^{\prime} u^{*}+P_{3}$ with $P_{3}>0$ since all the terms of $m_{11}$ are positive. Thus, from expression (3.2), one has

$$
m_{11}^{2} m_{22}-m_{21} m_{32} m_{13}=D f^{\prime} u^{*}\left[a\left(2 u^{*}+v^{*}\right)-\varphi\right]-g f^{\prime} u^{*} a\left(2 u^{*}+v^{*}\right)+P_{3} m_{22} .
$$

Then,

$$
A=(D-g) f^{\prime} u^{*} a\left(2 u^{*}+v^{*}\right)+D_{u} D f^{\prime} u^{*}+P_{3} m_{22} .
$$

In the case 2, our Hypothesis 2.2 implies that $g\left(S^{*}\right)<g\left(\lambda_{u}\right)<f\left(\lambda_{u}\right)=D_{u} \leq D$ for all $\left.S^{*} \in\right] \lambda_{v}, \lambda_{u}[$. Therefore, $A>0$. On the other hand, we can write $m_{11}^{2}=D g^{\prime} v^{*}+P_{4}$ with $P_{4}>0$. From expression (3.2) of $a_{23}$ and $m_{33}$, one has $a_{23}=m_{33}+\psi$. Therefore,

$$
m_{11}^{2} m_{33}-m_{31} a_{23} m_{12}=(D-f) g^{\prime} v^{*} m_{33}-f g^{\prime} v^{*} \psi+P_{4} m_{33} .
$$

Since $f\left(S^{*}\right)<f\left(\lambda_{u}\right)=D_{u} \leq D$ for all $\left.S^{*} \in\right] \lambda_{v}, \lambda_{u}[$, then

$$
B=(D-f) g^{\prime} v^{*} m_{33}+(D-f) g g^{\prime} v^{*}+f g^{\prime} v^{*} D_{v}+P_{4} m_{33}>0 .
$$

Thus, in the second case when $\lambda_{v}<\lambda_{u}$, we can conclude that $c_{4}>0$ for all $\left.S^{*} \in\right] \lambda_{v}, \lambda_{u}[$.

Appendix B. Parameters used in numerical simulations. All the values of the parameters

\begin{tabular}{|c|c|c|c|c|c|c|c|c|c|}
\hline Parameter & $\begin{array}{l}m_{1} \\
\left(h^{-1}\right)\end{array}$ & $\begin{array}{l}k_{1} \\
(g / l)\end{array}$ & $\begin{array}{l}m_{2} \\
\left(h^{-1}\right)\end{array}$ & $\begin{array}{l}k_{2} \\
(g / l)\end{array}$ & $\begin{array}{l}a \\
(l / h / g)\end{array}$ & $\begin{array}{l}b \\
\left(h^{-1}\right)\end{array}$ & $\begin{array}{l}D \\
\left(h^{-1}\right)\end{array}$ & $\begin{array}{l}D_{u} \\
\left(h^{-1}\right)\end{array}$ & $\begin{array}{l}D_{v} \\
\left(h^{-1}\right)\end{array}$ \\
\hline $\begin{array}{l}\text { Figures } 4 \text { to } 11 \\
\text { Figure } 12\end{array}$ & 5 & 2 & 5 & 3 & 4 & 2 & 0.1 & 3.35 & $\begin{array}{l}1.1 \\
0.09\end{array}$ \\
\hline Figure 13 & 4.5 & 1 & 3 & 2.7 & 2 & 3 & 3.5 & 3 & 2 \\
\hline Figure 14 & 60 & 0.5 & 0.6 & 20 & 0.01 & 0.01 & 50 & 50 & 0.2 \\
\hline Figures 15 and 16 & 4.5 & 1.7 & 4 & 1.5 & 2 & 0.8 & 3.2 & 3 & 1.6 \\
\hline Figure 17 & 20 & 1.5 & 2 & 2.7 & 1.2 & 3 & 47 & 15 & 1 \\
\hline Figure 18 & 4 & 2 & 3 & 1.5 & 2 & 3 & 3 & 3 & 2 \\
\hline
\end{tabular}
values used in numerical simulations are provided in the following Table.

TABLE 1

Parameter values used for (1.4) when the growth rates $f$ and $g$ are given by (4.1).

Acknowledgments. The authors would like to thank one anonymous reviewer for interesting comments and suggestions that improved the content of this work. We thank Jérôme Harmand for valuable discussions we had about this work. We thank the financial support of the Euro-Mediterranean research network TREASURE (http://www.inra.fr/treasure).

\section{REFERENCES}

[1] A. Ding and M.J. Hounslow and C.A. Biggs, Population balance modelling of activated sludge flocculation: Investigating the size dependence of aggregation, breakage and collision efficiency, Chem. Eng. Sci., 61 (2006), pp. 63-74, https://doi.org/10.1016/j.ces.2005.02.074.

[2] O. Bernard, Z. Hadj-Sadok, D. Dochain, A. Genovesi, and J.-P. Steyer, Dynamical model development and parameter identification for an anaerobic wastewater treatment process, Biotechnol. Bioeng., 75 (2001), pp. 424-438, https: //doi.org/10.1002/bit.10036.

[3] M. P. Boer, B. W. Kooi, and S. A. L. M. Kooijman, Food chain dynamics in the chemostat, Math. Biosci., 150 (1998), pp. 43-62, https://doi.org/10.1016/S0025-5564(98)00010-8.

[4] R. Fekih-Salem, Modèles mathématiques pour la compétition et la coexistence des espèces microbiennes dans un chémostat, PhD thesis, University of Montpellier 2 and University of Tunis el Manar, 2013, https://tel. archives-ouvertes.fr/tel-01018600.

[5] R. Fekih-Salem, J. Harmand, C. Lobry, A. Rapaport, and T. Sari, Extensions of the chemostat model with flocculation, J. Math. Anal. Appl., 397 (2013), pp. 292-306, https://doi.org/10.1016/j.jmaa.2012.07.055.

[6] R. Fekih-Salem, C. Lobry, and T. Sari, A density-dependent model of competition for one resource in the chemostat, Math. Biosci., 286 (2017), pp. 104-122, https://doi.org/10.1016/j.mbs.2017.02.007. 
[7] R. Fekih-Salem, A. Rapaport, and T. Sari, Emergence of coexistence and limit cycles in the chemostat model with flocculation for a general class of functional responses, Appl. Math. Modell., 40 (2016), pp. 7656-7677, https: //doi.org/10.1016/j.apm.2016.03.028.

[8] R. Freter, Mechanisms that control the microflora in the large intestine, in Human Intestinal Microflora in Health and Disease, (D. Hentges, ed. ), Academic Press, New York, 1983.

[9] R. Freter, H. Brickner, and S. Temme, An understanding of colonization resistance of the mammalian large intestine requires mathematical analysis, Microecol. Ther., 16 (1986), pp. 147-155.

[10] B. Haegeman, C. Lobry, and J. Harmand, Modeling bacteria flocculation as density-dependent growth, AIChE J., 53 (2007), pp. 535-539, https://doi.org/10.1002/aic.11077.

[11] B. Haegeman and A. Rapaport, How flocculation can explain coexistence in the chemostat, J. Biol. Dyn., 2 (2008), pp. 1-13, https://doi.org/10.1080/17513750801942537.

[12] J. Harmand, C. Lobry, A. Rapaport, and T. Sari, The Chemostat: Mathematical Theory of Microorganism Cultures, vol. 1, Wiley, Chemical Engineering Series, Chemostat and Bioprocesses Set, 2017.

[13] J. Harmand, A. Rapaport, D. Dochain, and C. Lobry, Microbial ecology and bioprocess control: Opportunities and challenges, J. Process Control, 18 (2008), pp. 865-875, https://doi.org/10.1016/j.jprocont.2008.06.017.

[14] IWA Task Group on Biofilm Modeling, Mathematical modeling of biofilms, IWA publishing, 2006.

[15] D. Jones, H. V. Kojouharov, D. Le, and H. Smith, Bacterial wall attachment in a flow reactor, SIAM J. Appl. Math., 62 (2002), pp. 1728-1771, https://doi.org/10.1137/S0036139901390416.

[16] D. Jones, H. V. Kojouharov, D. Le, and H. Smith, Bacterial wall attachment in a flow reactor: mixed culture, Can. Appl. Math. Quart., 10 (2002), pp. 111-137.

[17] D. Jones, H. V. Kojouharov, D. Le, and H. Smith, The Freter model: A simple model of biofilm formation, J. Math. Biol., 47 (2003), pp. 137-152, https://doi.org/10.1007/s00285-003-0202-1.

[18] D. A. Jones and H. Smith, Microbial competition for nutrient and wall sites in plug flow, SIAM J. Appl. Math., 60 (2000), pp. 1576-1600, https://doi.org/10.1137/S0036139998341588.

[19] B. W. Kooi and M. P. Boer, Chaotic behaviour of a predator-prey system in the chemostat, DCDIS, Series B: Applications and Algorithms, 10 (2003), pp. 259-272.

[20] Y. A. Kuznetsov, Elements of Applied Bifurcation Theory, Third edition. Springer, New York, 2004.

[21] C. Lobry and J. Harmand, A new hypothesis to explain the coexistence of $n$ species in the presence of a single resource, C. R. Biol., 329 (2006), pp. 40-46, https://doi.org/10.1016/j.crvi.2005.10.004.

[22] C. Lobry and F. Mazenc, Effect on persistence of intra-specific competition in competition models, Electron. J. Diff. Eqns., 125 (2007), pp. 1-10.

[23] C. Lobry, F. Mazenc, and A. Rapaport, Persistence in ecological models of competition for a single resource, C. R. Acad. Sci. Paris, Ser. I, 340 (2005), pp. 199-204, https://doi.org/10.1016/j.crma.2004.12.021.

[24] C. Lobry, A. Rapaport, and F. Mazenc, Sur un modèle densité-dépendant de compétition pour une ressource, C. R. Biol., 329 (2006), pp. 63-70, https://doi.org/10.1016/j.crvi.2005.11.004.

[25] S. Marsili-Libelli and S. Beni, Shock load modelling in the anaerobic digestion process, Ecol. Model., 84 (1996), pp. 215232, https://doi.org/10.1016/0304-3800(94)00125-1.

[26] S. S. Pilyugin and P. Waltman, The simple chemostat with wall growth, SIAM J. Appl. Math., 59 (1999), pp. 1552-1572, https://doi.org/10.1137/S0036139997326181.

[27] A. Rapaport, Properties of the chemostat model with aggregated biomass, Euro. J. of Appl. Math., 29 (2018), pp. 972990, https://doi.org/10.1017/S0956792518000141.

[28] T. Sari and R. Fekih-Salem, Analysis of a model of flocculation in the chemostat, in Proceedings of the 8th conference on Trends in Applied Mathematics in Tunisia, Algeria, Morocco, 2017, pp. 75-80, https://indico.math.cnrs.fr/ event/1335.

[29] T. Sari and J. Harmand, A model of a syntrophic relationship between two microbial species in a chemostat including maintenance, Math. Biosci., 275 (2016), pp. 1-9, https://doi.org/10.1016/j.mbs.2016.02.008.

[30] T. Sari and M. J. Wade, Generalised approach to modelling a three-tiered microbial food-web, Math. Biosci., 291 (2017), pp. 21-37, https://doi.org/10.1016/j.mbs.2017.07.005.

[31] S. Shen, G. C. Premier, A. Guwy, and R. Dinsdale, Bifurcation and stability analysis of an anaerobic digestion model, Nonlinear Dyn., 48 (2007), pp. 391-408, https://doi.org/10.1007/s11071-006-9093-1.

[32] J. Sieber, A. Rapaport, S. Rodrigues, and M. Desroches, A method for the reconstruction of unknown non-monotonic growth functions in the chemostat, Bioprocess Biosyst Eng, 36 (2013), pp. 1497-1507, https://doi.org/10.1007/ s00449-013-0912-8.

[33] H. L. Smith and P. Waltman, The Theory of the Chemostat: Dynamics of Microbial Competition, Cambridge University Press, 1995.

[34] E. D. Stemmons and H. L. Smith, Competition in a chemostat with wall attachment, SIAM J. Appl. Math., 61 (2000), pp. 567-595, https://doi.org/10.1137/S0036139999358131.

[35] D. N. Thomas, S. J. Judd, and N. Fawcett, Flocculation modelling: a review, Water Res., 33 (1999), pp. 1579-1592, https://doi.org/10.1016/S0043-1354(98)00392-3.

[36] M. Wade and R.W. Pattinson and N.G. Parker and J. Dolfing, Emergent behaviour in a chlorophenol-mineralising threetiered microbial 'food web', J. Theor. Biol., 389 (2016), pp. 171-186, https://doi.org/10.1016/j.jtbi.2015.10.032.

[37] A. Xu and J. Dolfing and T. P. Curtis and G. Montague and E. Martin, Maintenance affects the stability of a two-tiered microbial 'food chain'?, J. Theor. Biol., 276 (2011), pp. 35-41, https://doi.org/10.1016/j.jtbi.2011.01.026. 